OPEN ACCESS

Edited by:

Nadia Bianchi-Berthouze, University College London, United Kingdom

Reviewed by: Katarzyna Wac, Université de Genève, Switzerland Andrea Kleinsmith, University of Maryland, Baltimore County, United States

${ }^{*}$ Correspondence:

Jun $\mathrm{Hu}$ j.hu@tue.n

Specialty section: This article was submitted to Human-Media Interaction, a section of the journal Frontiers in ICT

Received: 25 July 2017 Accepted: 13 August 2018 Published: 07 September 2018

Citation:

Yu B, Funk M, Hu J, Wang $Q$ and Feijs $L$ (2018) Biofeedback for

Everyday Stress Management: A

Systematic Review. Front. ICT 5:23.

doi: $10.3389 /$ fict.2018.00023

\section{Biofeedback for Everyday Stress Management: A Systematic Review}

\author{
Bin Yu, Mathias Funk, Jun Hu*, Qi Wang and Loe Feijs \\ Department of Industrial Design, Eindhoven University of Technology, Eindhoven, Netherlands
}

Background: Mainly due to an increase in stress-related health problems and driven by recent technological advances in biosensors, microelectronics, computing platform, and human-computer interaction, ubiquitous physiological information will potentially transform the role of biofeedback in clinical treatment. Such technology is also likely to provide a useful tool for stress management in everyday life. The aim of this systematic review is to: (1) Classify biofeedback systems for stress management, with a special focus on biosensing techniques, bio-data computing approaches, biofeedback protocol, and feedback modality. (2) Review ways of evaluating approaches to biofeedback applications in terms of their effectiveness in stress management.

Method: A systematic literature search was conducted using keywords for "Biofeedback" and "Stress" within the following databases: PubMed, IEEE Xplore, ACM, and Scopus. Two independent reviewers were involved in selecting articles.

Results: We identified 103 studies published between 1990 and 2016, 46 of which met our inclusion criteria and were further analyzed. Based on the evidence reviewed, HRV, multimodal biofeedback, RSP, HR, and GSR appear to be the most common techniques for alleviating stress. Traditional screen-based visual displays remain the most common devices used for biofeedback display. Biofeedback applications are usually assessed by making both physiological and psychological measurements.

Conclusions: This review reveals several challenges related to biofeedback for everyday stress management, such as facilitating user's perception and interpretating the biofeedback information, the demand of ubiquitous biosensing and display technologies, and field evaluation in order to understand the use of biofeedback in everyday environments. We expect that various emerging $\mathrm{HCl}$ technologies could be used to address these challenges. New interaction designs as well as biofeedback paradigms can be further explored in order to improve the accessibility, usability, comfort, engagement with, and user experience of biofeedback in everyday use.

Keywords: biofeedback, stress management, relaxation, human-computer interaction, physiological computing

\section{INTRODUCTION}

\section{Chronic Stress and Health}

Stress is both a biological and a psychological response. It occurs when a situation is perceived to be challenging or threatening (i.e., meeting a work deadline or facing a speeding car). And the stress responses are mainly mediated by the human "stress system," which involves the amygdala, hypothalamus, autonomic nervous system (ANS), glands, and organs (Chrousos and Gold, 1992). 
When our brain perceives a stimulus as a stressor, it sends a distress signal to the hypothalamus, which then activates the sympathetic branch of ANS, sending signals to the adrenal glands (Porges, 1995). The hormone epinephrine is pumped into the bloodstream and acts on the target organs, speeding up the heartbeat and breathing, stiffening the muscles, and causing sweating. The combination of these reactions is known as the "fight-or-flight" response, which enables us to react quickly to life-threatening situations and help us fight off threats or flee to safety. Acute stress is transient, beneficial, and even vital in many cases. When a stressful situation passes, the parasympathetic branch of ANS is activated, acting as a "brake" to dampen the stress responses and help to re-establish homeostasis.

Unfortunately, this "brake" might fail to operate when our body overreacts to some chronic stressors, such as long-term work pressure. When the brain continuously perceives the situation as stressful, always-on "fight-or-flight" responses may put the ANS off balance and cause the responsiveness of our stress system to further deteriorate. The cumulative effects of chronic stress often degrade performance in work (Scott et al., 2006). Physiologically, long-term activation of adrenal glands can release excess cortisol (a stress hormone) which disrupts various bodily processes and disturbs homeostasis (Chrousos, 2009). Elevated cortisol level puts high-stress individuals at an increased risk of numerous health problems, including anxiety, depression (Burke et al., 2005), immune dysregulation (Padgett and Glaser, 2003), heart disease, hypertension (Esler and Kaye, 2000), and diabetes (Lloyd et al., 2005).

Keeping stress in an "optimal zone" is good both for work performance and overall health. Optimal responsiveness of the stress system is also essential for regulating healthy emotions in social interactions and a sense of well-being. This is why various techniques have been developed for coping with stress and these have been used to help people maintain homeostasis and autonomic balance. For instance, listening to relaxing music can reduce cortisol levels and help people recover from periods of stress (Khalfa et al., 2003). Research by Alvarsson et al. (2010) suggests that stress recovery will be faster and more complete when people are exposed to colors and sound stimuli recorded in natural environments. Tang et al. (2009) documented evidence showing that short-term meditation practice may improve the balance of ANS and that mindfulness practices deliver several positive benefits, including decreased anxiety and increased focus and improved mood (Smith, 2014). As suggested by Streeter et al. (2012), yoga stimulates an underactive parasympathetic nervous system and helps to correct an imbalance of ANS.

\section{Biofeedback for Stress Management}

Biofeedback is a powerful tool for stress management and relaxation training. It enables individuals to learn how to regulate their physiological activities in order to restore or maintain autonomic balance (Brown, 1977; Frank et al., 2010; Saha et al., 2015). As shown in Figure 1, a typical biofeedback loop consists of four components: a biosensing unit, a data transfer unit, a data processing unit, and a feedback unit. The biosensors monitor specific physiological activities, and immediately present related information back to the user with an external audiovisual display. Biofeedback techniques can help users improve selfawareness of internal states and learn self-regulation skills to manipulate specific physiological functions in a healthy direction. In clinical applications, biofeedback techniques often serve as a particular intervention or addition to cognitive and behavioral therapy. For instance, biofeedback devices are widely used to treat anxiety disorders (Reiner, 2008) or reduce psychological stress during an early postpartum period (Kudo et al., 2014). In such cases, biofeedback interventions are usually performed with the assistance of a well-trained therapist who introduces the biofeedback instrument, explains the procedure, interprets the meaning of feedback, and instructs the patient to use the feedback to achieve an optimal state.

Nowadays, in addition to clinical applications, the embodiment and modality of biofeedback have been broadened beyond traditional desktop settings. Advanced biosensors enable the data collection to be unobtrusive and wearable. The innovations in interaction design are improving the acceptance, usability, and user experience of biofeedbackassisted learning/training. Physiological computing can be completed on various computing platforms such as laptops, mobile phones, and wearable devices. As a result, biofeedback systems become increasingly portable, usable, and affordable, and the physiological information is now readily available in our everyday lives. With new interactive multimedia, biofeedback is no longer restricted to on-screen graphic displays, but is becoming increasingly diversified for different contexts, such as through tangible interaction (Ishii, 2008), peripheral interaction (Bakker et al., 2015), shape-changing display (Rasmussen et al., 2012), ambient display (Ishii et al., 1998), and musical interfaces (Gaye et al., 2003). Now, biofeedback techniques are more than a treatment for medical disorders; they are becoming a learning tool that average people can use to cope with everyday stress.

\section{The Objective of This Review}

Biofeedback applications have been widely reviewed in medical journals in terms of evidence and protocols. It is understood that biofeedback can effectively treat various stress-related and stress-induced disorders. Related reviews have mainly focused on evidence, medical applications, approaches, and practices of biofeedback. Some summarize and report the principles and practices of biofeedback in clinical applications (Blanchard et al., 1974; Basmajian, 1981). Some provide in-depth overviews of biofeedback techniques for medical treatments, such as headache disorders (Nestoriuc et al., 2008), anxiety disorders (Moore, 2000), hypertension (Greenhalgh et al., 2010), chronic pain (Turk et al., 1979), and stroke rehabilitation (Glanz et al., 1997). Yet no reviews have evaluated and discussed the biofeedback systems for everyday stress management, especially in relation to humaninformation interaction.

In this paper, we present a systematic review of the studies that have used biofeedback techniques for everyday stress management. The review summarizes the last 25 years of research on biofeedback applications for non-medical stress management. Based on this review, we hope to identify the research gaps in everyday biofeedback design as well as design opportunities. In particular, we aim to (1) classify the biofeedback systems, 


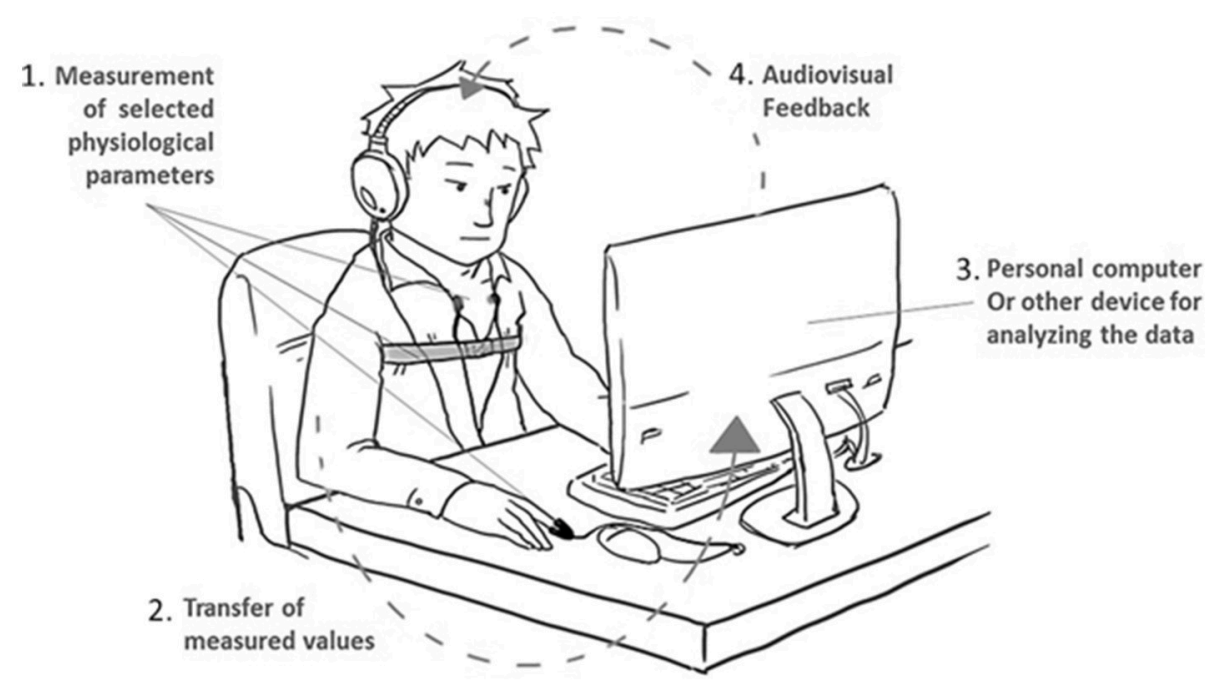

FIGURE 1 | Biofeedback is a closed-loop system that translates bio-signals into audiovisual displays.

with the emphasis on biosensing techniques, bio-data computing approaches, biofeedback protocols, and feedback modalities, and (2) inventory approaches taken to evaluate biofeedback applications in terms of their effectiveness for stress management.

\section{METHODOLOGY}

\section{Data Sources and Search Strategies}

We searched for relevant studies in the following electronic databases: PubMed, IEEE Xplore, ACM, and Scopus. To seek out articles, we selected papers that address two aspects: "Biofeedback" and "Stress Management." The following search strategy was applied in the PubMed database. MeSh (Medical Subject Heading) terms (the bold terms below) and their synonyms and spelling variations were used in several combinations and modified for other databases.

\#1 Biofeedback (biofeedback OR bio-feedback OR "augmented feedback" OR "sensory feedback" OR "sensory augmentation" OR "proprioceptive feedback" OR "auditory feedback" OR "audio feed-back" OR audio-feedback OR "visual feedback" OR "audiovisual feedback" OR "audiovisual feedback" OR "somatosensory feedback" OR "tactile feedback" OR "vibrotactile feedback" OR "vibratory feedback" OR "multimodal feedback")

\#2 Stress ("stress reduction" OR "stress management" OR "coping with stress" OR "stress relief” OR "stress intervention" OR distress OR anxiety OR meditation OR relaxation)

\#3 (1 AND 2)

\section{Selection Criteria}

Biofeedback was defined as measuring an individual's physiological activities and providing concurrent feedback about these activities to an individual. Stress management refers to any activity that helps to control, regulate, or reduce chronic stress responses that are not caused by diseases or traumas.
We only considered studies that applied biofeedback for stress management in general populations for non-therapeutic purposes. Articles published from 1990 to 2016 were reviewed.

The following exclusion criteria were applied to narrow down the set of relevant studies further. Firstly, regarding the applications of biofeedback, this review focuses on biofeedback techniques for average users in everyday use. For this reason, articles about biofeedback applications for therapeutic purposes or as a treatment for severe stress disorders were excluded. For instance, we excluded reports about using biofeedback for treating the stress that is caused by trauma (e.g., PTSD) (Lande et al., 2010), physical or mental illnesses (Reiner, 2008). Secondly, regarding the outcome evaluations, the studies were only considered if they used at least one objective measure of the effect of biofeedback on stress reduction. Studies that only describe the development of the system were excluded. Thirdly, articles were excluded if they were too general or too theoretical; for instance, those that presented a general biofeedback diagram or a design framework were excluded (e.g., Matuszek and Rycraft, 2003). Also, review articles, non-English publications, abstracts, tech reports, or other unpublished materials were excluded.

\section{Selection Procedures}

The search was performed on January 8th, 2017. The titles and abstracts of the results obtained by the database search were screened by two independent reviewers (BY \& QW). After titleabstract screening and a check for duplicates, we created an initial pool of articles. In the second filtering, these full-text articles were then analyzed and evaluated independently by the reviewers. In the case of discrepancies between the two reviewers, a third reviewer decided whether the article should be included. During this process, related studies cited in the articles were progressively included in our final database. 


\section{RESULTS}

\section{Search Results}

An overview of the results in the various stages of the selection process is visualized in Figure 2. The initial literature search yielded a total of 2,540 articles that were relevant to this review. After title and abstract screening and winnowing out duplicates and off-topic studies, 102 studies remained. The full papers of the remaining 102 studies were assessed to select those primary studies in biofeedback that directly related to everyday stress management.

Based on the stated exclusion criteria, 45 of the 102 publications were eligible for review. An additional article was retrieved from the reference and added to the database. Common reasons for exclusion included a lack of evaluation, the absence of a system description (e.g., Al Rihawi et al., 2014), or studies related to treatment of stress caused by a disease or trauma. In other cases, when the authors published several studies on the same research initiative, we only included their most recent research as long as it satisfied the inclusion criteria. Each study in the final selection $(n=46)$ was reviewed and information about the biofeedback techniques (e.g., measured bio-signals, biosensors), feedback displays (e.g., form-giving and interactive medium), stress management approaches, evaluation methods, and experiment design was extracted and summarized (see Table 1).

\section{Biofeedback Techniques}

Based on the content of biofeedback-physiological information-the biofeedback systems can be categorized into five types, as summarized in Figure 3. In some studies,
RSA (Respiratory Sinus Arrhythmia) biofeedback that presents successive IBI (Inter-Beat-Interval) data is regarded as a separate type of biofeedback. As the IBI data reflects heart rate variability (HRV) and assists users in regulating their respiration, in our review, RSA biofeedback is regarded as a particular type of HRV biofeedback. So, for the convenience of discussion, it is included in the HRV biofeedback group. Biofeedback systems that measure and present multiple types of physiological information are referred to as multimodal biofeedback systems.

\section{HRV Biofeedback (19 Studies)}

Heart rate variability (HRV) biofeedback (19/46) is the most common single-modal biofeedback technique for stress management. Figure 4 summarizes a typical diagram of an HRV biofeedback system. The input can be Electrocardiogram (ECG) signals or Blood Volume Pulse (BVP) signals. The content of feedback can be grouped as three types: IBI data, HRV indices, and a combination of both.

ECG signals reflect the amplified heart's electrical potential and they are measured by electrodes attached to an individual's limbs and the surface of the chest. BVP signals reflect the blood volume changes caused by the contraction of the heart. They are typically obtained by using a photoplethysmogram (PPG) sensor which illuminates the skin and detects changes in light absorption. ECG signals are often considered to be more accurate than BVP signals when calculating the inter-beat interval (IBI) because, compared to the curved peak of the pulse wave, the sharp upward spike of QRS wave could be detected more accurately by software algorithms. Several studies (Johnston and Mendelson, 2005) have verified that when the recordings are taken during a resting state (sitting still and quietly), the IBI

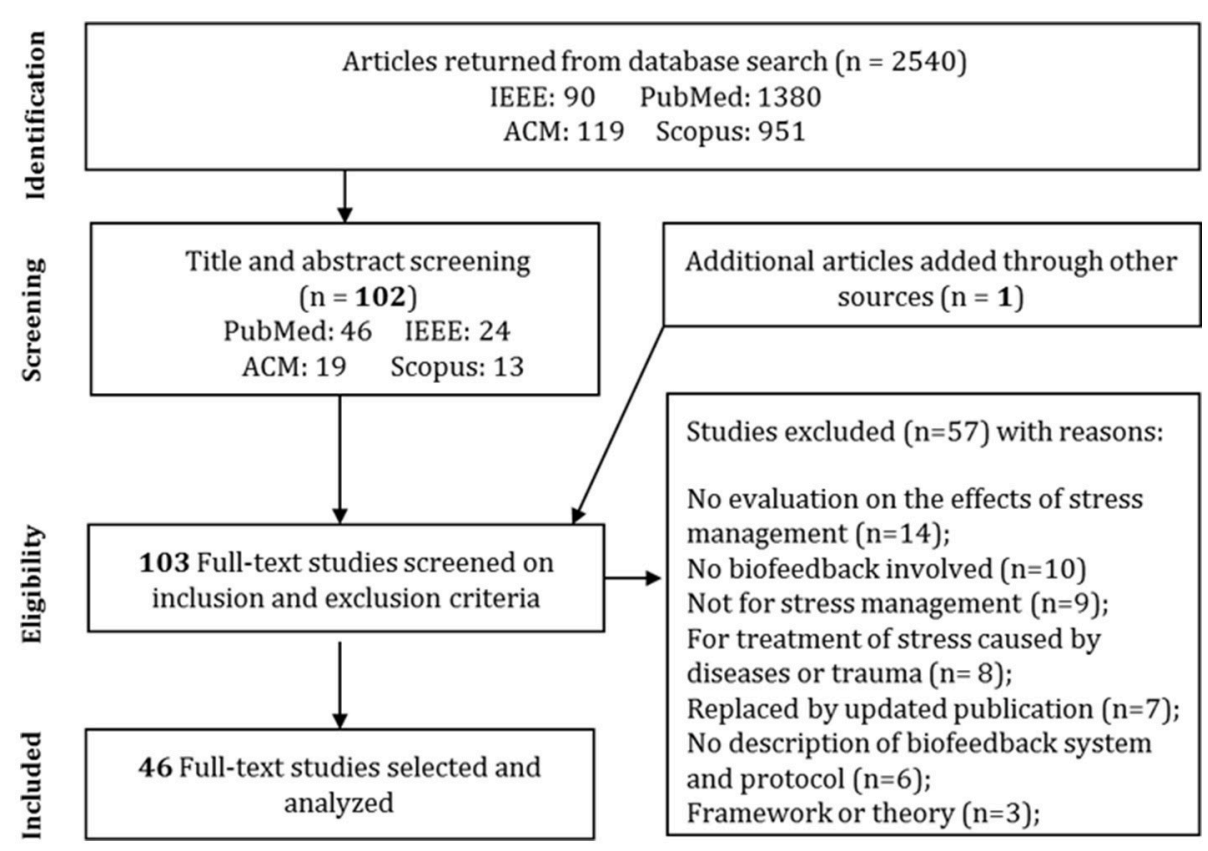

FIGURE 2 | Prisma (Moher et al., 2009) flowchart of the results of the literature search. 


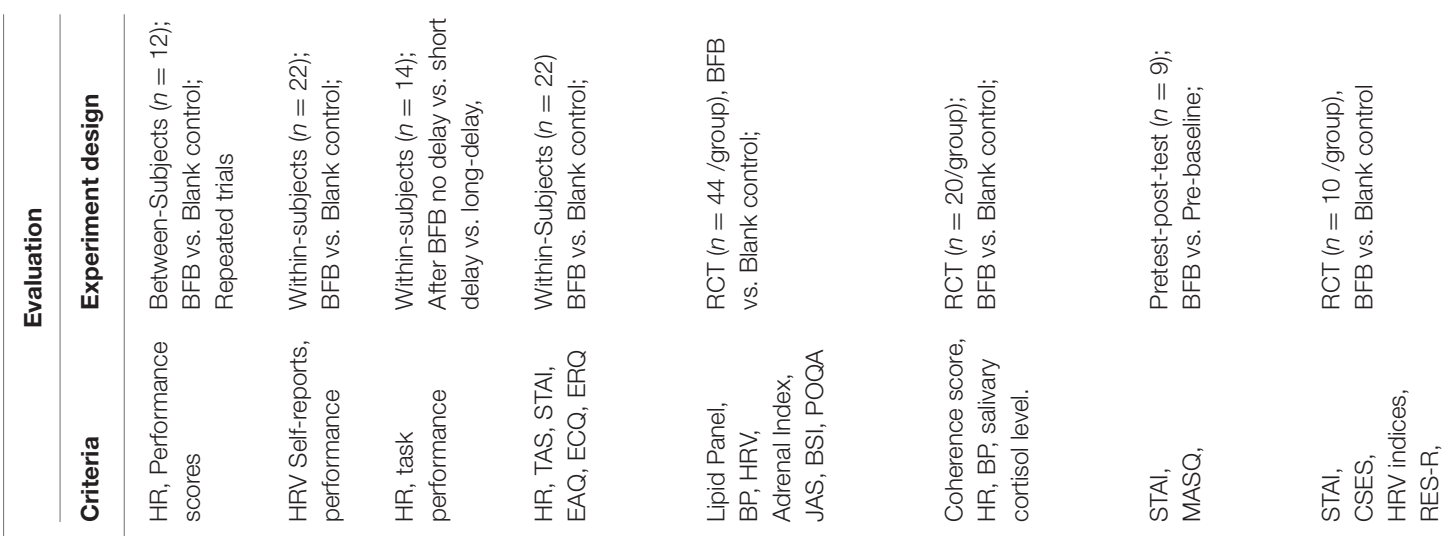

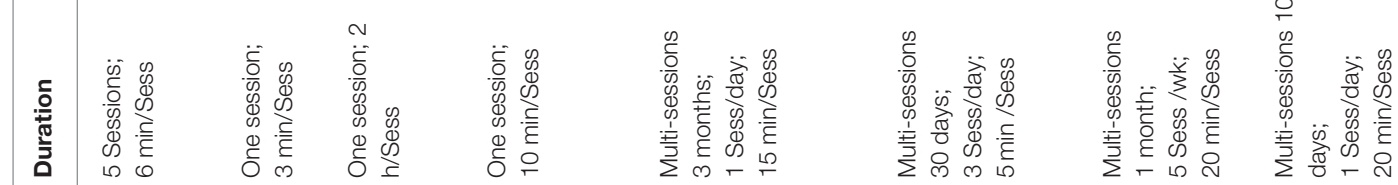

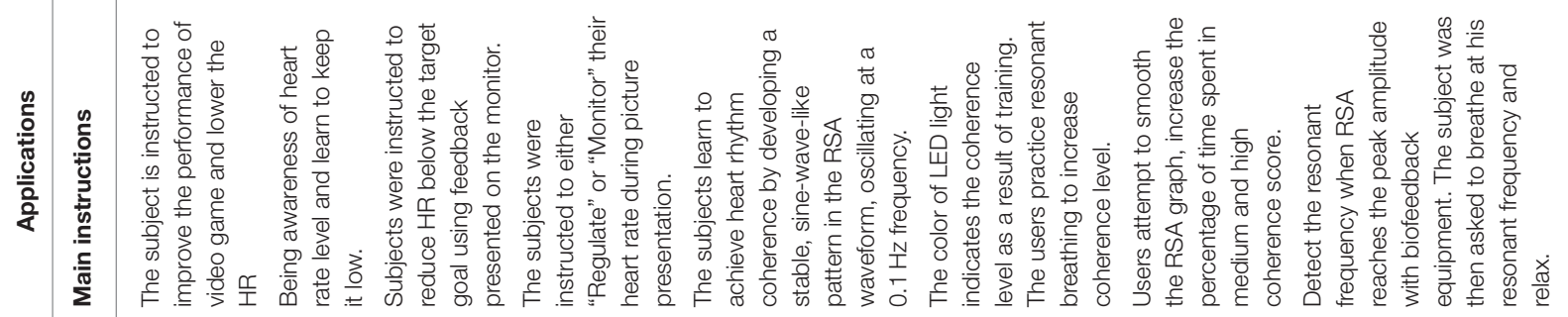

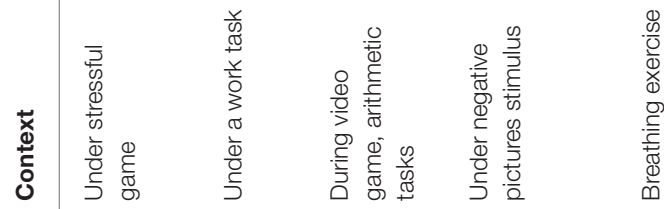

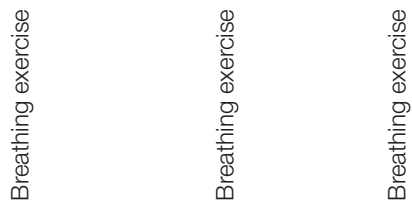

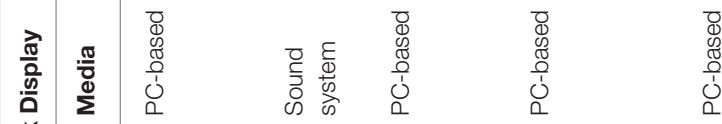

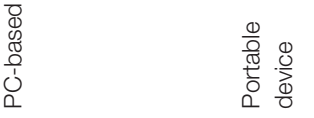

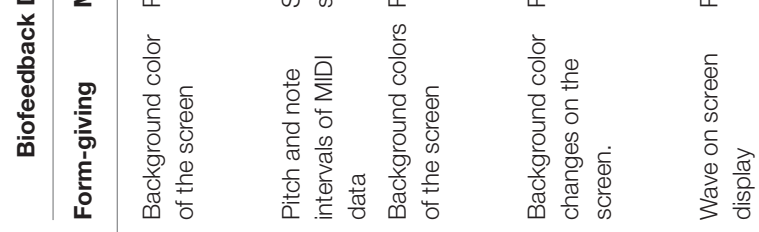

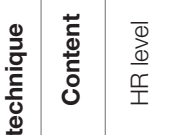

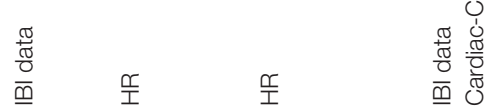

D
$\mathbb{8}$
$\mathbb{\pi}$
0
0
0
0

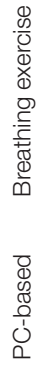

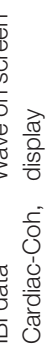

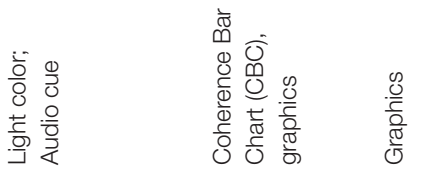

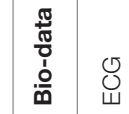

O

ভ্

$\sum_{i}^{n}$

ᄃ

ᄃำ

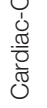

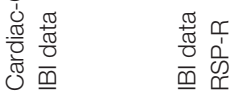

$\stackrel{\circ}{\Sigma}$

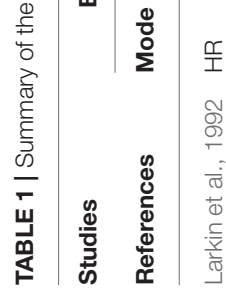

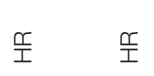

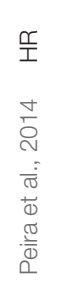

꼼

高

$\sum_{i}^{n} \quad \sum_{i}^{n}$

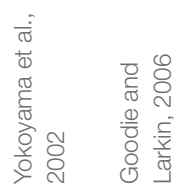

폼

卒竞 


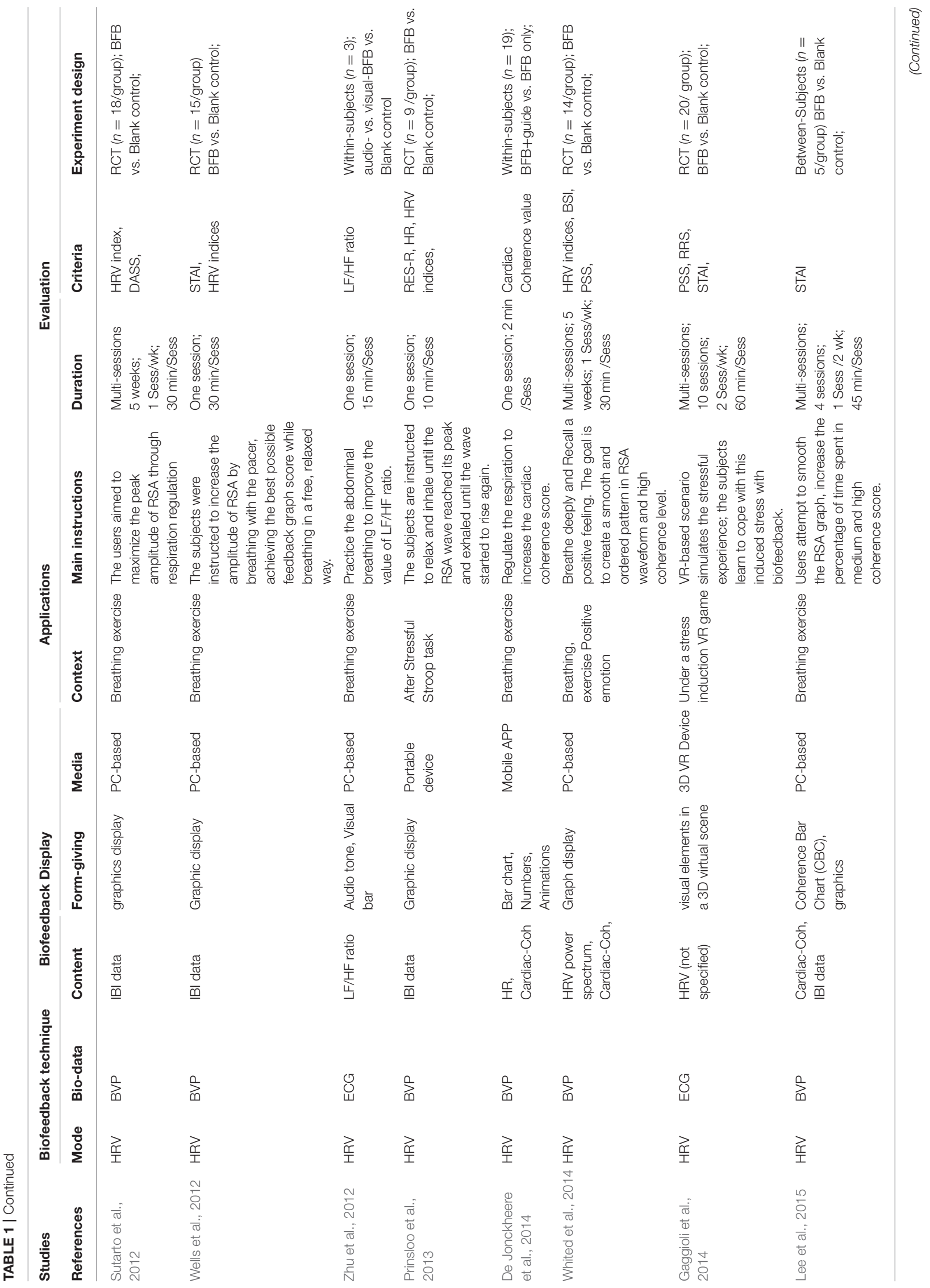




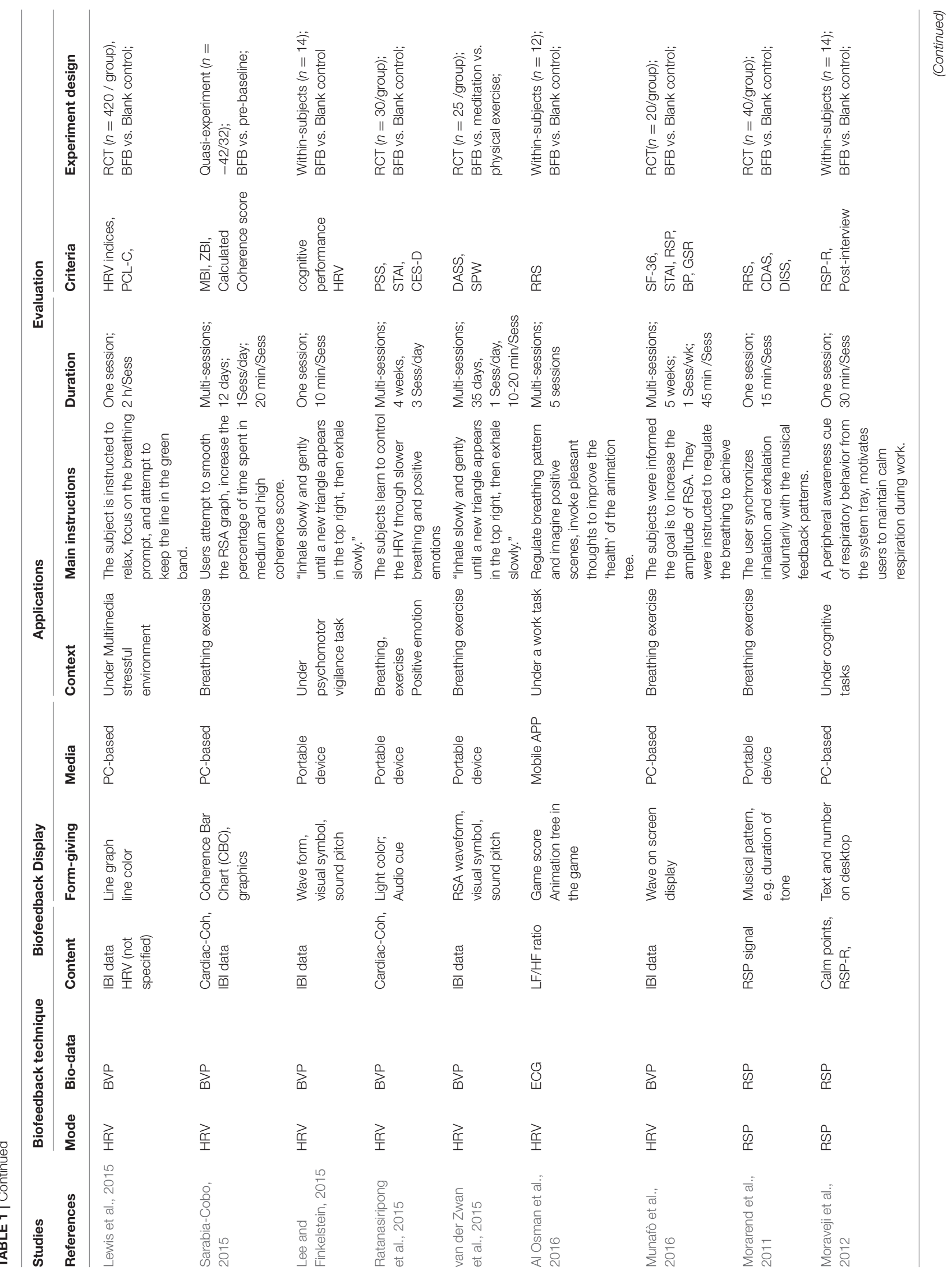




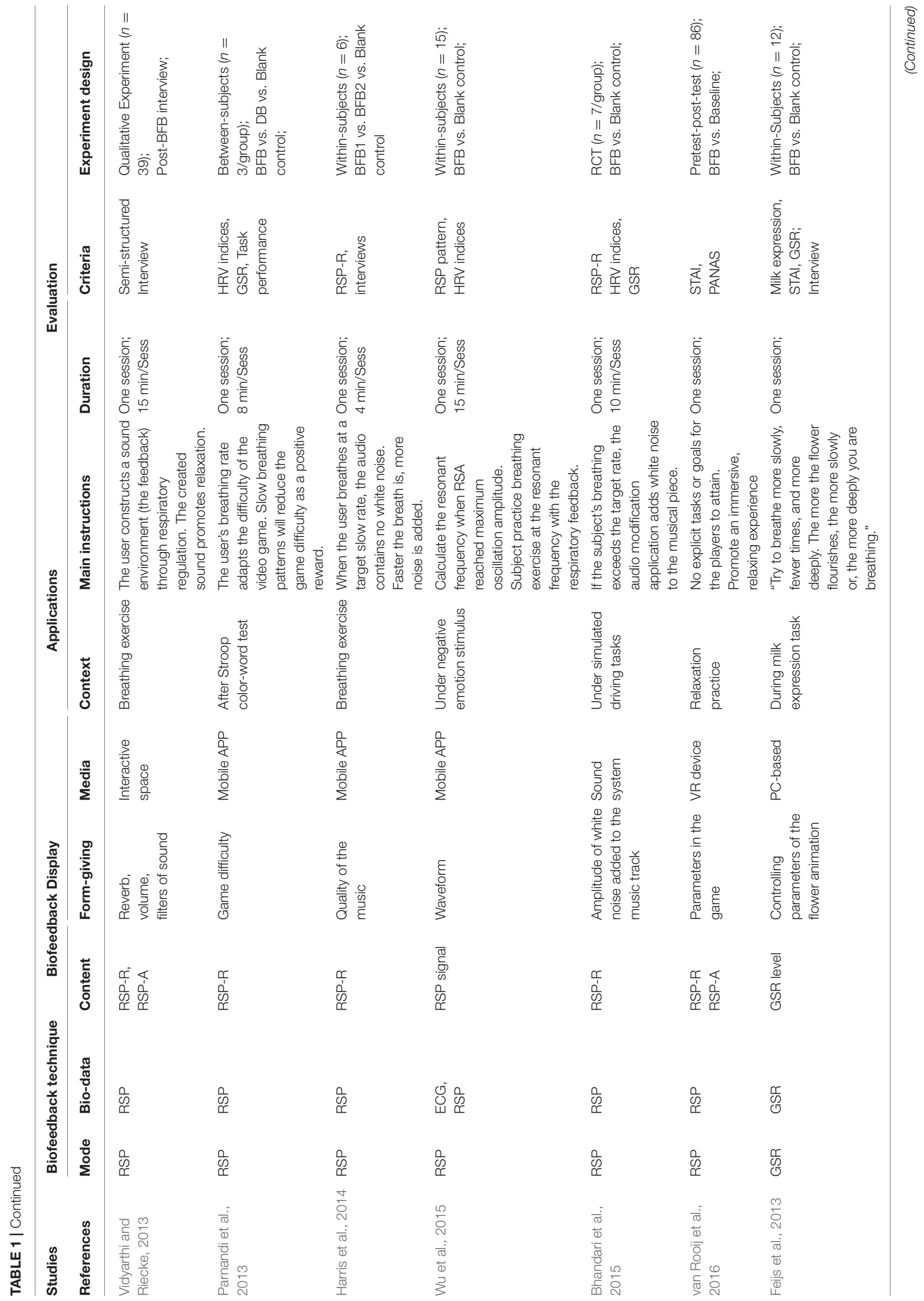




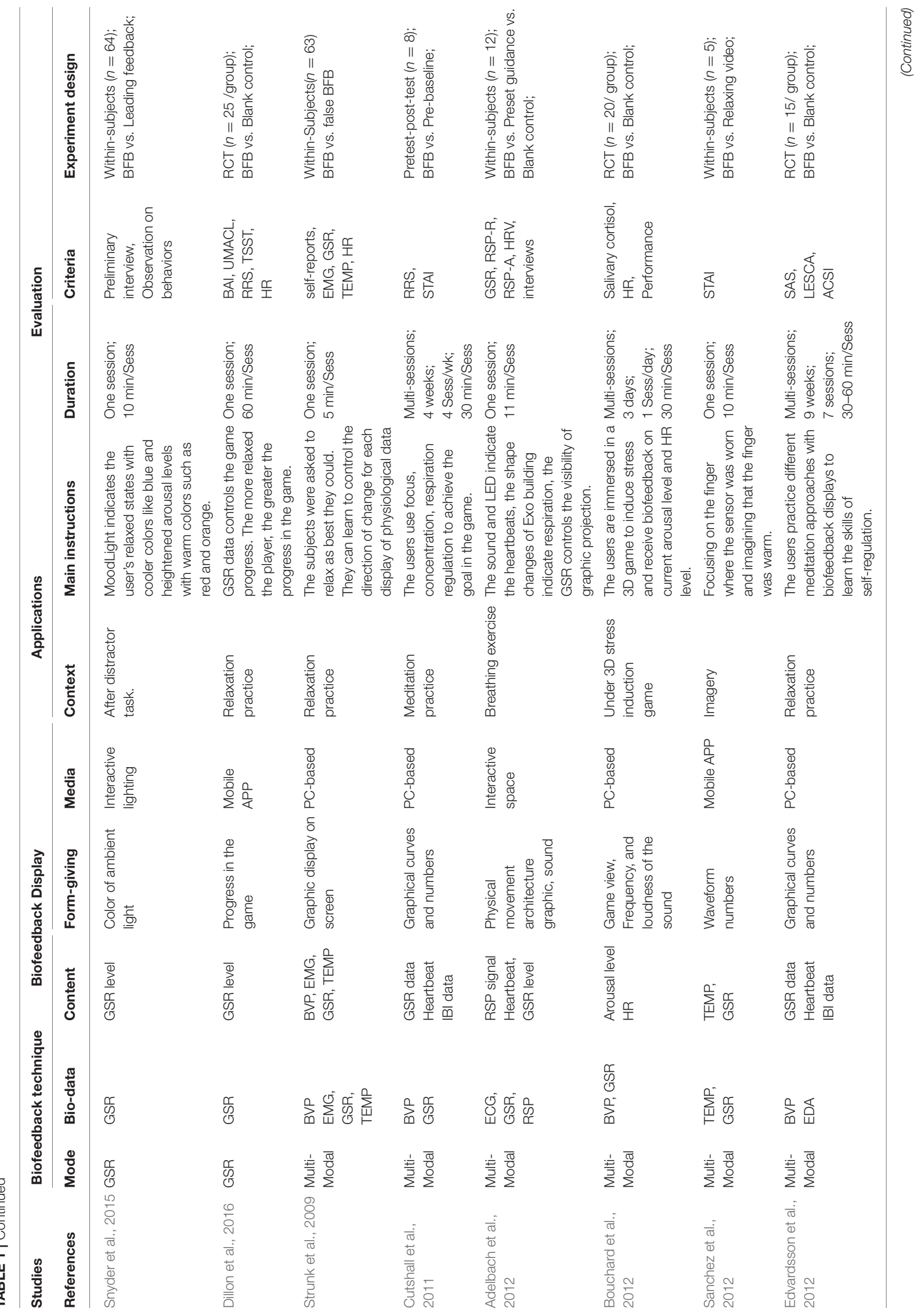




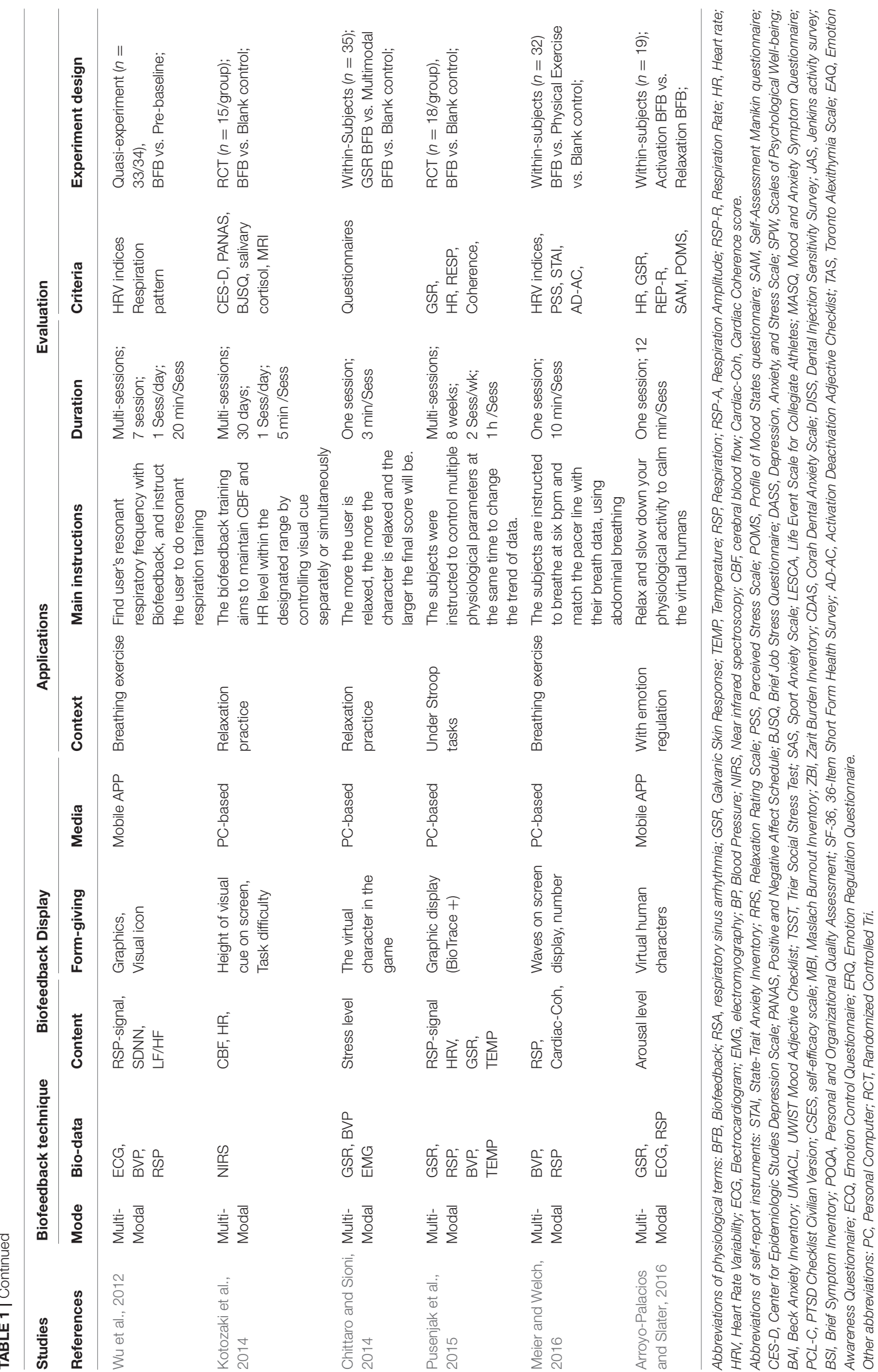




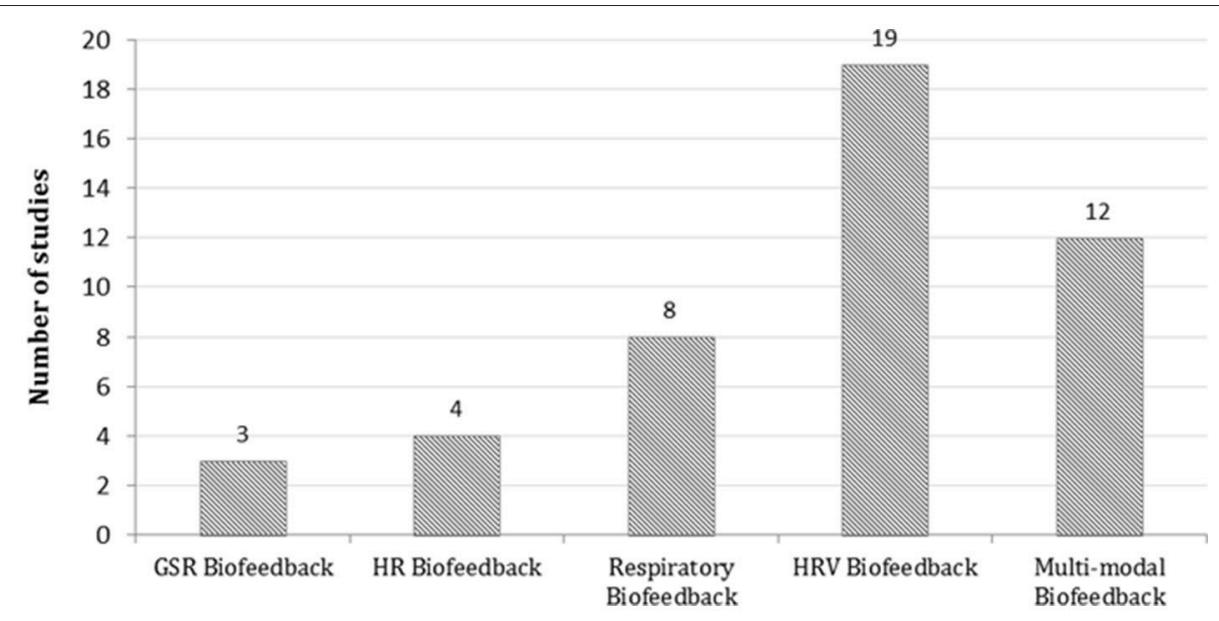

FIGURE 3 | The distribution of biofeedback techniques for stress management.

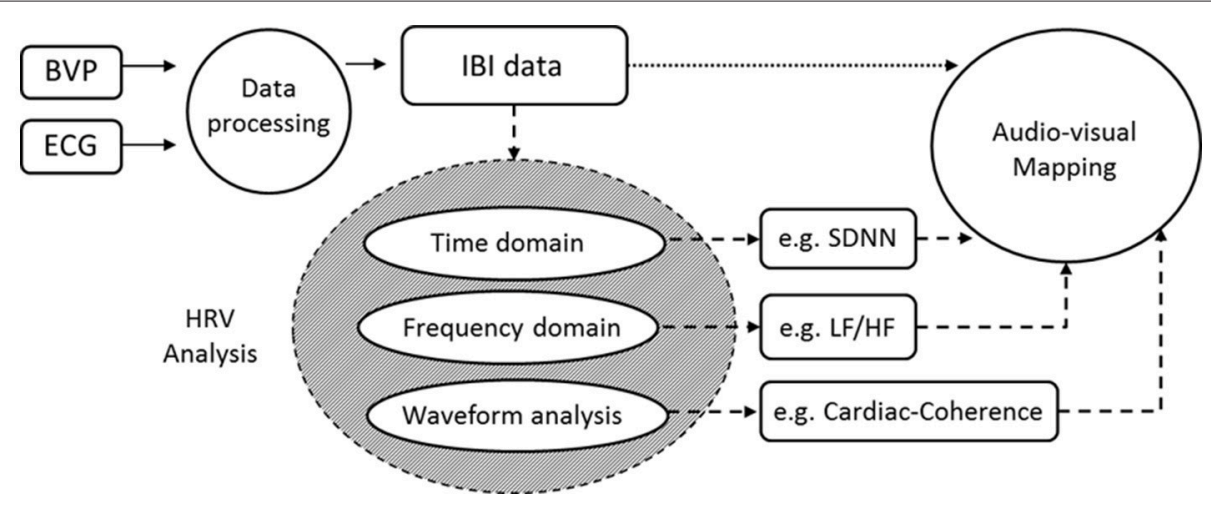

FIGURE 4 | A typical diagram of an HRV biofeedback system whose inputs can be BVP or ECG signals and output can be IBI and HRV indices.

calculated from ECG and BVP are highly correlated due to the improved peak detection from the pulse wave. PPG sensors have been recognized as a non-invasive and cost-effective method for measuring IBI and these are widely used in personal HRV biofeedback devices, such as emWave (Lemaire et al., 2011; Ratanasiripong et al., 2015), Wild Divine (Cutshall et al., 2011; Edvardsson et al., 2012), and StressEraser (Lee and Finkelstein, 2015).

Inter-Beat-Interval (IBI) is the time difference between two beat pulses. Heart rate varies in synchrony with respiration; the IBI is shortened during inspiration and prolonged during expiration (Lehrer et al., 2000). This physiologic phenomenon is referred to as Respiratory Sinus Arrhythmia (RSA). The IBI can be modulated by regulating respiration (i.e., resonant breathing) into a stable sine-wave-like pattern, through which the heart rate will achieve maximum oscillation. The immediate feedback of IBI is often used to assist users in learning resonant respiration for relaxation. This type of HRV biofeedback is also referred to as RSA biofeedback or IBI biofeedback (dotted arrow in Figure 4). The IBI data is usually transformed directly into an audiovisual display.
HRV refers to variations in heart beat intervals (Sztajzel, 2004). In an HRV biofeedback system, IBI data can be further analyzed to give more HRV indices (see the dashed arrows in Figure 4). Three main HRV indices are typically used for biofeedback in the context of stress management, namely cardiac coherence, the ratio of low-frequency to high-frequency power (LF/HF ratio) and SDNN (standard deviation of IBI data). Cardiac coherence score/value is the most common HRV feature used for biofeedback (McCraty et al., 2009; Henriques et al., 2011; Lemaire et al., 2011; De Jonckheere et al., 2014; Whited et al., 2014; Ratanasiripong et al., 2015; Sarabia-Cobo, 2015). Cardiac coherence describes the level of resonance between heart rate variations and the respiration cycle. A key marker of high cardiac coherence is the development of a smooth, sine-wave-like pattern in IBI waveform, oscillating at a low frequency of about $0.1 \mathrm{~Hz}$ ( $10 \mathrm{~s}$ per cycle). This is why, in these studies, the cardiac coherence score is usually calculated by analyzing the IBI pattern. For instance, in De Jonckheere et al. (2014), cardiac coherence is calculated by comparing the oscillation frequency of IBI data with $0.1 \mathrm{~Hz}$. A long oscillation period (close to $10 \mathrm{~s}$ ) helps achieve high cardiac coherence. 
In biofeedback training involving regulating breathing, the heart rate varies in synchrony with respiration; i.e., the IBI is shortened during inhaling and prolonged during exhaling (Lehrer et al., 2000). When the user's IBI wave is modulated into a stable sine-wave-like pattern under "resonance breathing," the $\mathrm{LF} / \mathrm{HF}$ ratio and SDNN are increased to close to the maximum value. $\mathrm{LF} / \mathrm{HF}$ ratio and SDNN, as the index of HRV, are therefore often used as an outcome measure, indicating users' performance when regulating breathing (Zhu et al., 2012; Whited et al., 2014; Al Osman et al., 2016).

\section{HR Biofeedback (4 Studies)}

Four studies explored HR biofeedback for stress management. As with HRV biofeedback, the input into an HR biofeedback system is also the ECG or BVP signal. The output is the instantaneous heart rate, which can be calculated from the IBI data. Heart rate data alone are insufficient to indicate stress. HR biofeedback is typically used for training users to reduce heart rate response/reactivity to acute stresses and to improve cardiac control during a stressful task, such as performing calculations using computers (Larkin et al., 1992; Yokoyama et al., 2002; Goodie and Larkin, 2006), or viewing negative pictures (Peira et al., 2014).

\section{Respiratory (RSP) Biofeedback (8 Studies)}

Eight studies (Morarend et al., 2011; Moraveji et al., 2012; Parnandi et al., 2013; Vidyarthi and Riecke, 2013; Harris et al., 2014; Bhandari et al., 2015; Wu et al., 2015) reported using RSP biofeedback for coping with stress. Respiratory biofeedback systems measure and present breathing-related information (i.e., depth and frequency) to help users learn specific breathing skills for relaxation and stress relief. A typical diagram of RSP biofeedback is shown in Figure 5. In RSP biofeedback systems, the respiration data is usually measured by a belt-type stretch sensor which is attached to a user's thorax or abdomen measuring chest/abdomen movements (expansion/contraction). The raw data of respiration movements can be directly mapped onto audiovisual output for immediate feedback (dotted arrow in Figure 5). For instance, respiration data can directly manipulate the sound output in real time (Vidyarthi and Riecke, 2013) or control the size of a circle pattern on a screen (van Rooij et al., 2016). Furthermore, more respiratory parameters, such as respiration rate and depth, can be extracted from the raw data (dashed arrows in Figure 5). These respiratory parameters are often used to control or adjust feedback displays, e.g., controlling game interfaces (van Rooij et al., 2016), adapting the level of difficulty in games (Parnandi et al., 2013), and adjusting music qualities (Harris et al., 2014; Bhandari et al., 2015). In Morarend et al. (2011), the average of the inhale and exhale time is used to synthesize real-time musical patterns with differentiated "inspiration" and "expiration" sounds.

\section{GSR Biofeedback (3 Studies)}

Galvanic skin response (GSR) has also been described as electrodermal activity (EDA). In this review, we use a unified term for such biofeedback systems: GSR biofeedback. Three studies relate to the use of GSR biofeedback for stress management. GSR sensors measure the subtle changes in skin conductivity which indicate the variations in an individual's arousal levels via the autonomic nervous system. GSR feedback is usually about the trend in such changes. The changes in GSR data can be calculated by comparing the new GSR data to the average value of successive moving windows (Feijs et al., 2013; Dillon et al., 2016). The Least Means Squares (LMS) on successive windows of data can also be applied to determine the slope of the GSR curve (Snyder et al., 2015).

\section{Multimodal Biofeedback (12 Studies)}

Twelve studies relate to the use of multimodal biofeedback. A multimodal biofeedback system measures more than one type of bio-signal as input, and the output can be a parallel display of various physiological data or an indicator of stress or emotional state based on analysis of multiple data feeds. As shown in Table 2, we divide these multimodal biofeedback systems into three categories: dual-channel (primary and secondary), multichannel (parallel), and affective feedback.

In the first category, eight studies relate to the use of biofeedback systems that can provide two channels of biodata. Basically, one of them will serve as the primary feedback, indicating the main physiological activity that is being regulated by the user. This feedback helps users to learn to improve selfregulation skills through practice in controlling that activity. For instance, the GSR data are used for learning to regulate arousal (Bouchard et al., 2012; Sanchez et al., 2012), the IBI data (Cutshall et al., 2011; Edvardsson et al., 2012) and the RSP data are used for regulating breathing (Adelbach et al., 2012; Wu et al., 2012; Meier and Welch, 2016). The other bio-data are mainly used as secondary information, indicating the results of the biofeedback training. For instance, the HRV data in an IBI biofeedback system may indicate the results of relaxation training. In the second category, two of the 12 multimodal biofeedback studies (Strunk et al., 2009; Pusenjak et al., 2015) present four types of bio-data through a graphic display on the screen, so users could observe and control various physiological processes synchronously. In the third category, multiple bio-data are further analyzed into affective information, indicating the user's emotional state or stress level (Chittaro and Sioni, 2014; Arroyo-Palacios and Slater, 2016). This type of affective feedback facilitates users to learn and improve the skills of emotion regulation and stress management.

\section{Biofeedback Display}

We present the results of the biofeedback display from two points of view: form-giving and interactive medium. Form-giving is a specific phrase in interaction design. Here it refers to the embodiment of the biofeedback interface or the representation of biofeedback data. The form-giving of biofeedback may range from professional graphics to a casual game. In different sensory modalities, biofeedback can be presented through various interactive media: vision (e.g., screens, ambient lights, or head-mounted displays), hearing (e.g., speakers or headphones), haptics (e.g., vibrotactile actuators), or a combination of these techniques. 


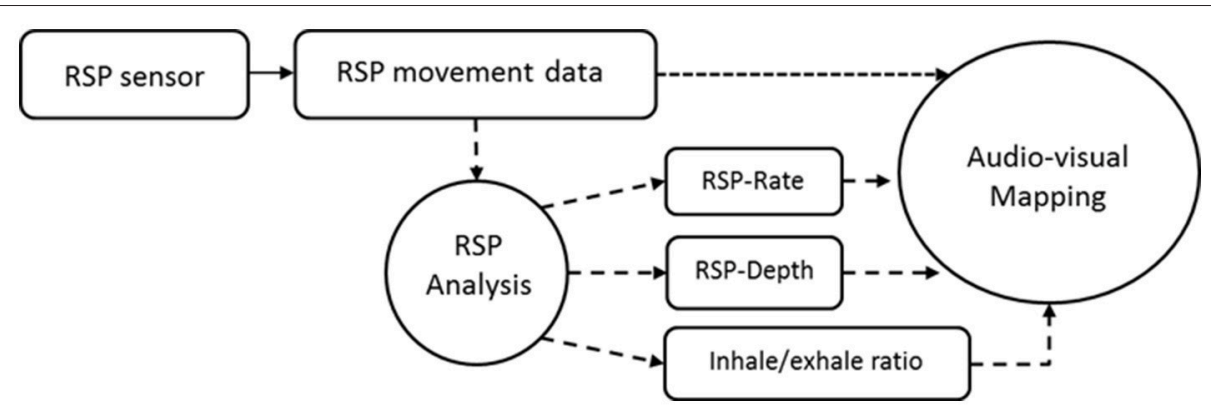

FIGURE 5 | A typical diagram of RSP biofeedback system whose outputs can be the raw data of respiration movements or respiratory parameters such as respiration rate and depth.

TABLE 2 | The main components of multimodal biofeedback.

\begin{tabular}{|c|c|c|}
\hline Type & $\begin{array}{l}\text { Biofeedback information } \\
\text { primary info }+ \\
\text { (secondary info) }\end{array}$ & Studies \\
\hline \multirow{4}{*}{$\begin{array}{l}\text { Dual-channel } \\
\text { (primary and } \\
\text { secondary) }\end{array}$} & GSR + (TEMP, HR) & $\begin{array}{l}\text { Bouchard et al., 2012; Sanchez } \\
\text { et al., } 2012\end{array}$ \\
\hline & $\mid \mathrm{BI}+(\mathrm{GSR})$ & $\begin{array}{l}\text { Cutshall et al., 2011; Edvardsson } \\
\text { et al., } 2012\end{array}$ \\
\hline & $\mathrm{RSP}+(\mathrm{HRV}, \mathrm{GSR})$ & $\begin{array}{l}\text { Adelbach et al., 2012; Wu et al., } \\
\text { 2012; Meier and Welch, } 2016\end{array}$ \\
\hline & $\mathrm{CBF}+(\mathrm{HR})$ & Kotozaki et al., 2014 \\
\hline $\begin{array}{l}\text { Multi-channel } \\
\text { (parallel) }\end{array}$ & HRV, EMG, GSR, TEMP & $\begin{array}{l}\text { Strunk et al., 2009; Pusenjak } \\
\text { et al., } 2015\end{array}$ \\
\hline Affective feedback & Stress or Emotion states & Arroyo-Palacios and Slater, 2016 \\
\hline
\end{tabular}

\section{Form-Giving}

As shown in Table 3, based on our results, we classified the biofeedback displays into three categories based on sensory modality: visual, audio, and a combination of both. These biofeedback displays were further classified by their form-giving, see Figure 6A.

Most studies (38/46) employed visual displays, in which wave diagrams, histograms, or direct displays of the data value were very common (e.g., Cutshall et al., 2011; Sanchez et al., 2012; Wu et al., 2015). Besides technical or medical graphics, biofeedback data can also be represented in the form of animation and games. For instance, HRV or GSR data are displayed with an animation of a living plant, such as a tree (Al Osman et al., 2016) or a flower (Feijs et al., 2013). Biofeedback data can also be mapped onto some visual elements in a relaxing nature scene, such as a meadow (De Jonckheere et al., 2014), a beach (Gaggioli et al., 2014), or an underwater environment (van Rooij et al., 2016). A virtual character is often used to express a user's stress level and emotional states (Chittaro and Sioni, 2014; Arroyo-Palacios and Slater, 2016). In addition, to improve the level of motivation and engagement in the training, biofeedback data can be used to modify the difficulty of a game, such as the Frozen
Bubble (Parnandi et al., 2013). Beyond screen-based displays, biofeedback can be presented by using ambient lights (Snyder et al., 2015).

In five studies, the biofeedback systems employed a singlemodal auditory display. These auditory displays take the form of music. The biofeedback data can be directly coupled to the pitch or to the intervals (between notes) of MIDI data to create music outputs (Yokoyama et al., 2002; Morarend et al., 2011). In other auditory designs, the quality of pre-selected music is modified by the biofeedback data in real time (Vidyarthi and Riecke, 2013; Harris et al., 2014; Bhandari et al., 2015). As auditory displays liberate users' eyes, these systems can be used at work (Yokoyama et al., 2002) and when engaged in demanding tasks such as driving (Bhandari et al., 2015).

Six studies relate to the use of biofeedback systems that provide a combination of audio and visual displays. Biofeedback data are presented simultaneously in both auditory and visual modalities to enhance the user's perception and improve the usability of the interface. For instance, in StressEraser, the on-screen graphics combined with an audio clue to present IBI data (Lee and Finkelstein, 2015; van der Zwan et al., 2015). In EmWave2, the indicator light works together with an audio cue to show coherence between respiration and heart rhythm (Lemaire et al., 2011; Ratanasiripong et al., 2015). The auditory and visual modalities can also be used separately to present various types of bio-data. For instance, Adelbach et al. (2012) developed a biofeedback-driven adaptive architecture, in which respiration is presented by movements of the physical structure, heart rate is indicated by sounds, and GSR data is presented by graphics projected on the ceiling.

\section{Interactive Media}

Besides the form-giving, interactive media also play an important role in user interaction with biofeedback systems. Based on the dominant interactive medium in the user interface, the selected studies can be classified roughly into four categories, as shown in Figure 6B. Screen-based biofeedback systems are used in most of the studies. The biofeedback data are displayed on the screen of a personal computer $(n=24)$ or a mobile/portable device $(n=15)$. The ambient medium allows the users to receive biofeedback information through the surroundings in a physical 
TABLE 3 | The form-giving of the biofeedback systems.

\begin{tabular}{|c|c|c|}
\hline Sensory modality & Form-giving & Studies \\
\hline \multirow[t]{6}{*}{ Visual } & Graphic and numeric & $\begin{array}{l}\text { Larkin et al., 1992; Goodie and Larkin, 2006; McCraty et al., 2009; Strunk et al., 2009; Cutshall et al., 2011; Henriques } \\
\text { et al., 2011; Morarend et al., 2011; Edvardsson et al., 2012; Moraveji et al., 2012; Paul and Garg, 2012; Sanchez } \\
\text { et al., 2012; Sutarto et al., 2012; Wells et al., 2012; Wu et al., 2012, 2015; Prinsloo et al., 2013; Peira et al., 2014; } \\
\text { Whited et al., 2014; Lee et al., 2015; Lewis et al., 2015; Pusenjak et al., 2015; Sarabia-Cobo, 2015; Meier and Welch, } \\
\text { 2016; Munafò et al., } 2016\end{array}$ \\
\hline & Animations & Feijs et al., 2013; Al Osman et al., 2016 \\
\hline & Visual scene & Bouchard et al., 2012; De Jonckheere et al., 2014; Gaggioli et al., 2014; Dillon et al., 2016; van Rooij et al., 2016 \\
\hline & Virtual character & Chittaro and Sioni, 2014; Arroyo-Palacios and Slater, 2016 \\
\hline & Modified game & Parnandi et al., 2013; Kotozaki et al., 2014 \\
\hline & Ambient light & Snyder et al., 2015 \\
\hline Auditory & Musical expression & Yokoyama et al., 2002; Morarend et al., 2011; Vidyarthi and Riecke, 2013; Harris et al., 2014; Bhandari et al., 2015 \\
\hline Audio-visual & Combination & $\begin{array}{l}\text { Lemaire et al., 2011; Adelbach et al., 2012; Zhu et al., 2012; Lee and Finkelstein, 2015; Ratanasiripong et al., 2015; } \\
\text { van der Zwan et al., } 2015\end{array}$ \\
\hline
\end{tabular}
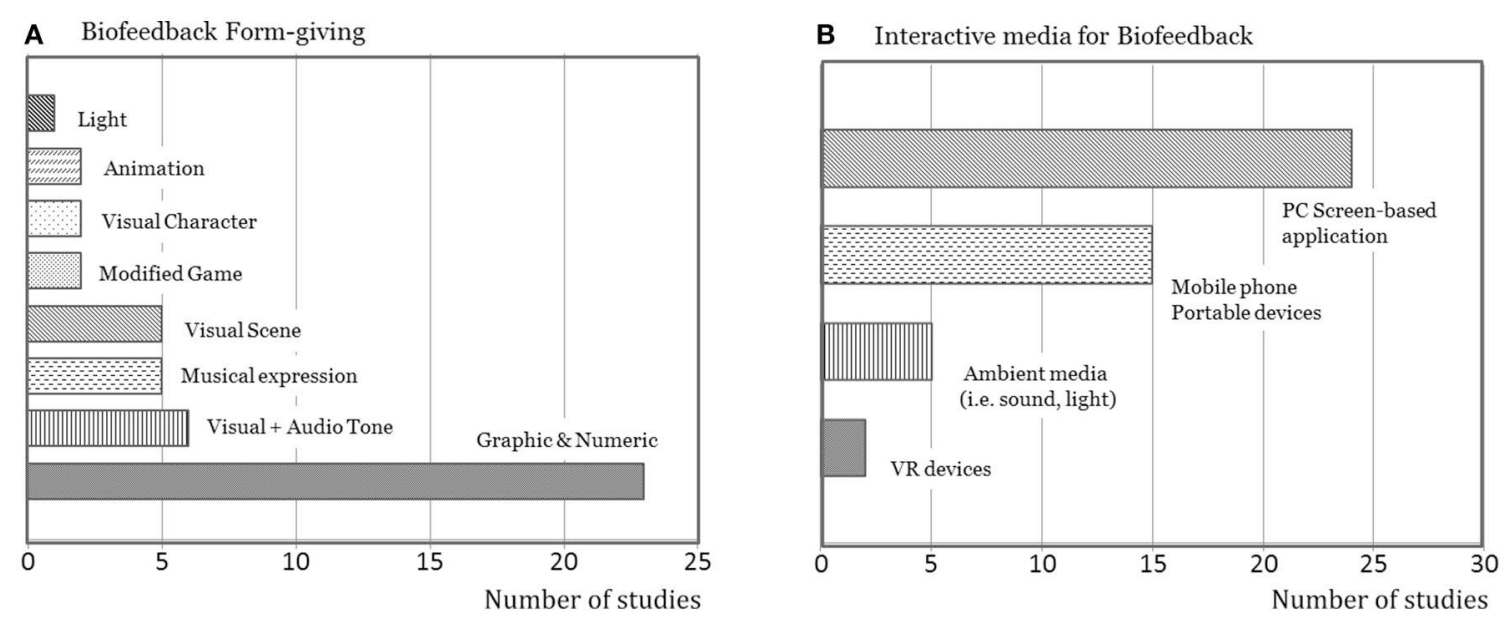

FIGURE 6 | Overview of biofeedback displays in terms of form-giving and interactive media (A). Most displays are graphic and numeric (B). PC-based biofeedback applications are most common.

environment. For instance, The GSR data can be displayed through changes in ambient light (Snyder et al., 2015). The respiration data can be presented by physical space that changes shape (Adelbach et al., 2012). In recent years, Virtual Reality (VR) devices have also been used in biofeedback displays in order to enhance immersive relaxing experiences (Gaggioli et al., 2014; van Rooij et al., 2016).

\section{Use Scenarios Involving Biofeedback Relaxation Training With Biofeedback}

Biofeedback techniques are widely used in relaxation training, such as resonant breathing, positive imagery, yoga, and meditation. In these biofeedback-assisted types of training, users typically sit quietly and focus on self-regulation practices using feedback. The biofeedback systems serve as a tool to facilitate the acquisition of particular self-regulation skills. In most studies, the RSP and HRV biofeedback are used to improve breathing skills, including abdominal breathing (e.g., Zhu et al., 2012; Meier and Welch, 2016), and resonant breathing (e.g., Lemaire et al., 2011;
Paul and Garg, 2012). Biofeedback has also been used in imagery relaxation (e.g., Sanchez et al., 2012), meditation (e.g., Cutshall et al., 2011), and psychotherapy designed to stimulate positive emotions (e.g., Whited et al., 2014).

\section{Stress Intervention With Biofeedback}

Besides assisting with relaxation training, biofeedback techniques are also used to improve users' resilience to acute stress as well as, negative emotional and mental stimuli. Typically, this type of biofeedback training is performed during or just after a simulated stressful experience, such as a stressful work task (e.g., Yokoyama et al., 2002; Al Osman et al., 2016), a mentally challenging task (e.g., Prinsloo et al., 2013), a negative multimedia stimulus (Goodie and Larkin, 2006; Lewis et al., 2015), or a stressful game (Bouchard et al., 2012). Here, the biofeedback helps to improve users' awareness of their stress and find a better (mostly personalized) approach to reducing or moderating stress responses, e.g., via activities designed to increase arousal (heart rate and skin conductance responses). 

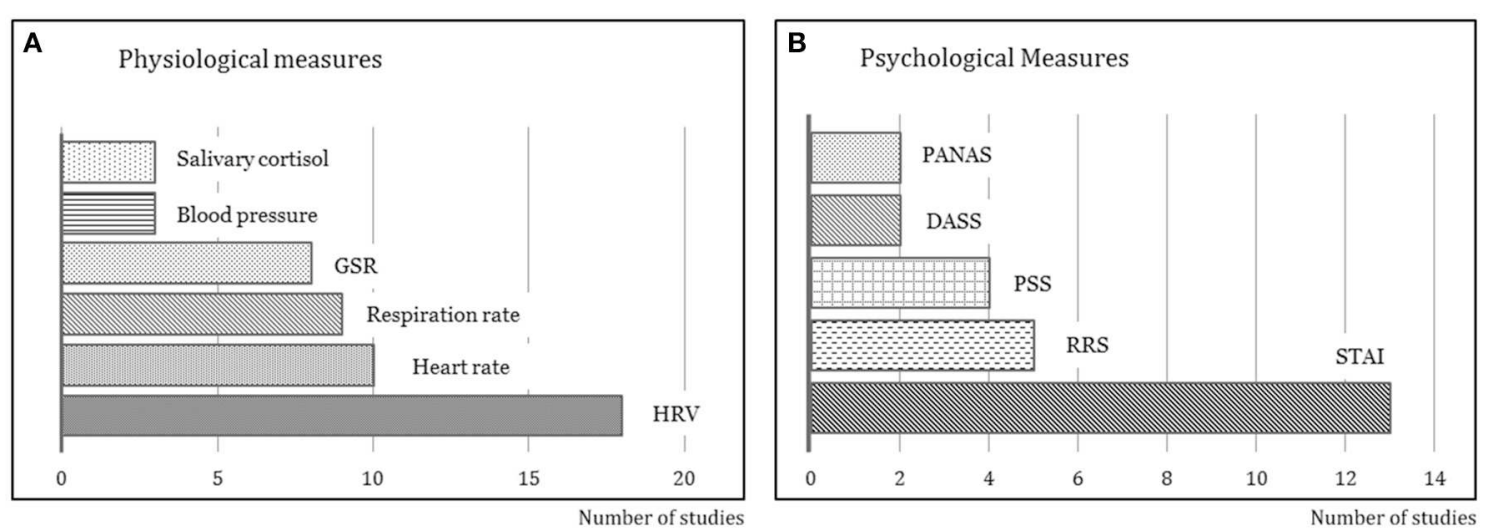

FIGURE 7 | The physiological and psychological measures used to evaluate biofeedback training for stress management (A). HRV is the most commonly used physiological measure (B). STAl is the most commonly used psychological measure.

\section{Evaluations of the Effectiveness of Biofeedback for Stress Reduction Experimental Design}

The evaluation of biofeedback training varied widely in the selected studies. These can be broadly divided into five categories, based on the approaches taken and the standards adopted: pretest post-test design, within-subjects design, between-subjects design, quasi-experiment design, and Randomized Controlled Trial (RCT). Three studies (Cutshall et al., 2011; Henriques et al., 2011; van Rooij et al., 2016) designed a pre-test posttest experiment to compare the metrics before and after the biofeedback training. A total of 18 studies used within-subjects design and 23 studies used between-subjects design (including 20 RCTs). In addition, two studies (Wu et al., 2012; Sarabia-Cobo, 2015) used a quasi-experimental design, and one study (Vidyarthi and Riecke, 2013) conducted a qualitative experiment through a post-test interview.

To investigate the effects of biofeedback training, a control group or a control condition is usually required for comparison. Most of the studies $(n=33)$ used a blank control group or condition, where the participants receive neither biofeedback nor other alternatives. In addition, biofeedback training can also be compared with other relaxation approaches, such as a watching a relaxing video (Sanchez et al., 2012), following a preset relaxation guidance (Adelbach et al., 2012; De Jonckheere et al., 2014; Snyder et al., 2015), receiving a fake biofeedback signal (Strunk et al., 2009), performing deep breathing (Parnandi et al., 2013), and doing meditation (van der Zwan et al., 2015), or physical exercise (Meier and Welch, 2016).

\section{Metrics}

Table 4 shows typical metrics used to assess the effectiveness of biofeedback for stress alleviation. These metrics include four types of measurements: physiological, psychological, performance, and relaxing experience.

As shown in Figure 7A, typical physiological measures include heart rate variability (HRV), (18 studies), heart rate (HR) (10 studies), respiration (RSP) (9 studies), and galvanic skin response (GSR) (8 studies). HRV is the physiological metric that is most commonly used. In an ambulatory recording, HRV can indicate the regulatory capacity of an individual facing a stressful situation or event. In short recordings (5-15 min), HRV is highly related to respiratory cycles. This is why it provides a better indication of the effectiveness of biofeedback for regulating breathing. HRV includes a series of parameters that are calculated from the IBI analysis in the time or frequency domain. The typical time domain parameters include SDNN, RMSSD ${ }^{1}$, and pNN50 ${ }^{2}$. In the frequency domain, the LF/HF ratio reflects the global sympathovagal balance and is therefore often used to indicate the balance of the autonomic nervous system.

In a stressful situation, the body's sympathetic nervous system is activated, which quickly increases heart rate, blood pressure, and respiration rate. So heart rate and respiration rate provide a simple and direct measurement of the body's stress responses. As shown in Table 4, ten studies measured the average heart rate, and nine studies measured respiration rate. The GSR signal consists of two main components: skin conductance level (SCL) and skin conductance responses (SCRs). SCL is also known as tonic level. It is continually changing and closely related to the autonomic regulation. SCRs show that the phasic responses ride on top of the tonic changes. SCRs change fast and are more sensitive to emotionally arousing stimuli and events. Most studies used the mean level of SCL as the GSR measure of autonomic regulation. One study (Bhandari et al., 2015) used SCRs as the GSR measure of arousal level.

As shown in Figure 7B, various self-report instruments have been developed and these are widely used as psychological measures to assess stress or anxiety levels, including STAI (13 studies), RRS (5 studies), PSS (4 studies), DASS (2 studies), and PANAS (2 studies). Table 4 lists typical self-report instruments from the selected studies. Thirteen studies relate to the use of the

\footnotetext{
${ }^{1}$ RMSSD: the square root of the mean of the squares of the successive differences between adjacent inter-beat intervals.

${ }^{2}$ pNN50, the proportion of NN50 divided by the total number of inter-beat intervals. NN50: the number of pairs of successive inter-beat-intervals that differ by more than $50 \mathrm{~ms}$.
} 
TABLE 4 | The measurements used in evaluation of biofeedback training.

\begin{tabular}{|c|c|c|}
\hline Measures & Instruments & Studies \\
\hline \multirow[t]{6}{*}{ Physiological measures } & $\operatorname{HRV}(n=18)$ & $\begin{array}{l}\text { Yokoyama et al., 2002; McCraty et al., 2009; Lemaire et al., 2011; Adelbach et al., 2012; Paul and Garg, 2012; Sutarto } \\
\text { et al., 2012; Wu et al., 2012, 2015; Zhu et al., 2012; Parnandi et al., 2013; Prinsloo et al., 2013; Whited et al., 2014; } \\
\text { Bhandari et al., 2015; Lee and Finkelstein, 2015; Lewis et al., 2015; Sarabia-Cobo, 2015; Al Osman et al., 2016; Meier } \\
\text { and Welch, } 2016\end{array}$ \\
\hline & $\operatorname{GSR}(n=8)$ & $\begin{array}{l}\text { Strunk et al., 2009; Adelbach et al., 2012; Feijs et al., 2013; Parnandi et al., 2013; Bhandari et al., 2015; Pusenjak } \\
\text { et al., 2015; Arroyo-Palacios and Slater, 2016; Munafò et al., } 2016\end{array}$ \\
\hline & $\mathrm{HR}(n=10)$ & $\begin{array}{l}\text { Larkin et al., 1992; Goodie and Larkin, 2006; Strunk et al., 2009; Lemaire et al., 2011; Bouchard et al., } 2012 \text {; Prinsloo } \\
\text { et al., 2013; Peira et al., 2014; Pusenjak et al., 2015; Arroyo-Palacios and Slater, 2016; Dillon et al., } 2016\end{array}$ \\
\hline & $\operatorname{RSP}(n=9)$ & $\begin{array}{l}\text { Adelbach et al., 2012; Moraveji et al., 2012; Wu et al., 2012, 2015; Prinsloo et al., 2013; Bhandari et al., 2015; } \\
\text { Pusenjak et al., 2015; Arroyo-Palacios and Slater, 2016; Munafò et al., } 2016\end{array}$ \\
\hline & $\mathrm{BP}(n=3)$ & McCraty et al., 2009; Lemaire et al., 2011; Munafò et al., 2016 \\
\hline & Salivary Cortisol $(n=3)$ & Lemaire et al., 2011; Bouchard et al., 2012; Kotozaki et al., 2014 \\
\hline
\end{tabular}

Psychological measures STAI $(n=13)$

Cutshall et al., 2011; Henriques et al., 2011; Paul and Garg, 2012; Sanchez et al., 2012; Wells et al., 2012; Feijs et al., 2013; Gaggioli et al., 2014; Peira et al., 2014; Lee et al., 2015; Ratanasiripong et al., 2015; Meier and Welch, 2016; Munafò et al., 2016; van Rooij et al., 2016

$\operatorname{RRS}(n=5) \quad$ Cutshall et al., 2011; Morarend et al., 2011; Gaggioli et al., 2014; Al Osman et al., 2016; Dillon et al., 2016

PSS $(n=4) \quad$ Gaggioli et al., 2014; Whited et al., 2014; Ratanasiripong et al., 2015; Meier and Welch, 2016

DASS $(n=2) \quad$ Sutarto et al., 2012; van der Zwan et al., 2015

PANAS $(n=2) \quad$ Kotozaki et al., 2014; van Rooij et al., 2016

Performance

Larkin et al., 1992; Yokoyama et al., 2002; Goodie and Larkin, 2006; Bouchard et al., 2012; Feijs et al., 2013; Parnandi et al., 2013; Lee and Finkelstein, 2015

User experience Interview

Adelbach et al., 2012; Moraveji et al., 2012; Feijs et al., 2013; Vidyarthi and Riecke, 2013; Chittaro and Sioni, 2014 ; Harris et al., 2014; Snyder et al., 2015

State-Trait Anxiety Inventory (STAI) as the measure of trait and state anxiety. STAI has 20 items for assessing trait anxiety and 20 items for state anxiety. The state anxiety sub-inventory (STAIS) can indicate perceived stress "at the moment." Five studies relate to the use of Relaxation Rating Scale (RRS) as a simple and fast instrument. RRS only requires the participant to rate his/her level of stress or relaxation on a Likert-type scale by circling the number that best described his/her current experience. It is therefore better suited to experiments that require repeated measurements or have a time limit.

Four studies relate to the use of Perceived Stress Scale (PSS) to measure the perception of stress. Because the questions in PSS ask about feelings and thoughts during the past month, it is more suitable for measuring the general stress level in one's life and the degree to which situations in life are appraised as stressful. Two studies relate to the use of DASS, and two relate to the use of PANAS. Depression, Anxiety, and Stress Scale (DASS) is designed to measure four related negative emotional states: depression, anxiety, tension, and stress. Each scale contains 14 items, which measure the average level of emotional states during the past week. Positive and Negative Affect Schedule (PANAS) focuses on the measurements of two mood scales: positive affect and negative affect. It consists of 20 adjectives which describe a type of mood; the respondent indicates the extent to which he/she has felt this way over the past week. Different from STAI and RRS, which measure "at-the-moment" stress feelings, PSS, DASS, and PANAS measure general stress or anxiety levels over the past week or month. This is why they are more suitable for evaluating the effects of a long-term biofeedback intervention.

In addition to the physiological and psychological measures, a user's work/task performance can also be measured to assess the effects of biofeedback. Performance measurements can produce a score of a mentally challenging task including calculation work (Yokoyama et al., 2002), an arithmetic task (Larkin et al., 1992; Goodie and Larkin, 2006), and a modified Stroop colorword test (Parnandi et al., 2013). The measurements can be specialized by tasks, such as a psychomotor vigilance task (Lee and Finkelstein, 2015), lactation yield (Feijs et al., 2013), or some behavioral indices assessed by a medical instructor (Bouchard et al., 2012). In addition, in seven studies, qualitative data about user experience were also collected through a follow-up interview and observations. The qualitative studies were designed to gain more insights into user experience and user opinions on interaction design. The interview data can be used to support the interpretation of quantitative data and provide more information about psychological states.

\section{Summary of Main Results}

This systematic review inventories the biofeedback techniques, feedback displays, use scenarios, and evaluation approaches in the selected studies. Based on the studies reviewed, we identified four types of single-modality biofeedback-HRV (41\%), RSP (17\%), HR (9\%), GSR (7\%), and multimodal biofeedback (26\%) - all designed to alleviate stress. Regarding biofeedback displays, traditional visual displays $(50 \%)$ are still the most 
common. However, an increasing number of playful and casual interfaces, such as visual animation and game (24\%), musical interfaces (24\%), and some ambient displays (2\%) have also been explored in recent years. Biofeedback displays still mainly depend on screen-based displays in PC (52\%) and mobile applications (33\%). New interactive media have gradually been explored for biofeedback, including virtual reality (4\%), ambient light (2\%), and adaptive architecture (2\%). The use of biofeedback techniques is broadly classified into two types: assistance with training in relaxation and intervention in a stressful task. The effects of biofeedback-assisted training are typically evaluated from a physiological perspective recording measures of HRV (39\%), HR (22\%), RSP (17\%), GSR (17\%), BP (7\%), and from a psychological point of view with self-report instruments such as STAI (28\%), RRS (11\%), PSS (9\%), and DASS (4\%).

\section{DISCUSSION}

Biofeedback can be used as a "mirror" to improve self-awareness about internal states, but also as a learning tool to acquire specific self-regulation skills. Based on this review, we found that an increasing number of casual and personal biofeedback systems have been designed for everyday stress management, which does not require a coach or a therapist. This has raised new demands for biofeedback systems in terms of biosensing, information display, HCI design, and evaluation.

\section{Discussion of Biofeedback Information}

Biofeedback information can be classified into two types: that reflecting a physiological process designed to assist in selfregulation (performance feedback) and that indicating the results of self-regulation training (result indices). For the first type, measured bio-signals are usually processed simply and presented immediately, as in the graphic display of IBI data (Sutarto et al., 2012; Prinsloo et al., 2013). Immediate feedback enables users to learn to regulate their physiological activities through practice in controlling the feedback in real time. This closedloop mechanism provides the basis for improving self-regulation skills through a process of trial and error. For the second type, the result indices, such as HRV features (Al Osman et al., 2016), arousal level (Snyder et al., 2015), and emotional states (Chittaro and Sioni, 2014), can be obtained by a sophisticated approach of physiological or affective computing. The feedback of results information informs users about their training results and the goals they have achieved (e.g., via a cardiac coherence score) and can also be used as a motivating factor to engage users in training.

Biofeedback systems may provide users with one or more types of information. From our review, it appears that unimodal biofeedback systems are more widely used in everyday stress management. Unimodal biofeedback systems monitor and present a specific physiological activity, such as respiration (Morarend et al., 2011), heart rate variability, (Prinsloo et al., 2013), and changes in EDA (Snyder et al., 2015). Users usually focus on a single self-regulation goal (e.g., achieving a sine-wave-like IBI wave) and practice a specific skill (e.g., resonant breathing) to achieve it more effortlessly. Because fewer biosensors are needed, unimodal biofeedback devices are usually portable and easy to operate (e.g., the mobile APP and emWave2 device). Multimodal biofeedback systems, such as $\mathrm{NeXus-10^{3 }}$ from MindMedia, can measure various physiological activities synchronously by using a wide range of biosensors. Such systems provide richer information, indicating both performance levels and the results of practicing self-regulation. For instance, the multimodal biofeedback system makes it possible to calculate the relationship between various physiological processes, such as the resonance score between HRV and respiration (Meier and Welch, 2016). However, it has also become clear from this review that multimodal biofeedback systems require new designs of information display to enhance user perception and understanding of multiple feedback information and also more advanced biosensing techniques to improve the usability and comfort of the biosensors.

\section{Discussion of Biosensing Techniques}

In this review, we found that most of the current biofeedback applications still use traditional biosensing techniques and approaches, such as using a photoplethysmogram (PPG) sensor clip on the fingertip to measure IBI (McCraty et al., 2009; Paul and Garg, 2012) or using a stretch sensor on the chest to measure respiration (Moraveji et al., 2012). We think this might be because physiological signals are prone to interference from body movements. Traditional biosensing approaches can efficiently avoid motion artifact and improve the signal-to-noise ratio. Although such biosensors and attachment approaches are acceptable in clinical applications, unobtrusive comfortable data collection is urgently required for everyday use. Especially when it comes to relaxation training, attaching multiple wired sensors restricts the user's posture, degrades the user experience or even may introduce new forms of stress.

Interestingly, many wearable contactless biosensors already exist, but these are mostly used for monitoring, and not yet for biofeedback. For instance, the Emaptica's E4 ${ }^{4}$ wristband offers an ambulatory recording of heart rate, electrodermal activity, and temperature. The Spire's Stone $e^{5}$ is a wearable device that monitors respiration via the expansion and contraction of the torso. Adib et al. (2015) uses a low power wireless signal to monitor the user's breathing and heart rate without body contact. Droitcour et al. (2001) developed a technology using a microwave radio to generate Doppler radar that senses breathing and heart rate wirelessly. Cho et al. (2017) explored using the new low-cost thermal camera attached to smartphone to track a user's breathing pattern, which can be further analyzed with the proposed deep learning model to automatically recognize the user's psychological stress level and cognitive load (Abdelrahman et al., 2017). Alongside these new biosensing technologies, traditional biosensors can still be used, but with new wearable designs to improve usability and comfort. For instance, the traditional PPG sensor can be integrated into an earphone (Poh et al., 2009) or a pair of glasses (Constant et al., 2015)

\footnotetext{
${ }^{3}$ NeXus-10, MindMedia: https://www.mindmedia.com/en/products/nexus-10mkii/

${ }^{4}$ Emaptica's E4, https://www.empatica.com/en-eu/research/e4/

${ }^{5}$ Spire's Stone: https://spire.io/pages/stone
} 
for unobtrusive heart rate monitoring. These mobile and noncontact sensors and newly-developed wearables are speeding up the application of biofeedback technology in an everyday context.

\section{Discussion of Biofeedback Display}

The presentation of biofeedback information may affect the perception and interpretation of information and also the user experience. Based on the studies reviewed, graphic user interfaces (GUI) are most commonly used to present information in a clear and precise manner. A simple graphic display (Munafò et al., 2016) and a pure auditory tone (Zhu et al., 2012; Lee and Finkelstein, 2015) have proven to be effective ways of delivering information. However, these basic visual or audio displays tend to be difficult to understand when first used and tend not to engage users in long-term use. In everyday use, such displays present a high barrier for non-specialist users and seem less engaging for relaxation training in non-clinical contexts, such as at home or in the workplace. This is why, in new interfaces, biofeedback data are presented through a richer form of interaction including casual games (Al Osman et al., 2016), metaphorical visualizations (Yu et al., 2017), and musical interfaces (Bhandari et al., 2015). For multimodal biofeedback systems, the concurrent feedback of multi-channel bio-data requires the investment of more effort in visualization and interface design. For instance, different data can be mapped onto various visual elements of the interface (De Jonckheere et al., 2014) or distributed to different sensory modalities of the display (e.g., a combination of sound, light, and changes in physical shape in Adelbach et al., 2012).

Biofeedback displays are not only an information "carrier" but also a stimulus that can influence the user experience. A biofeedback display can enhance relaxation but may also cause new forms of stress. For instance, in musical biofeedback interfaces (e.g., Lundqvist et al., 2009), the music signals not only convey information but also serve as a stimulus for promoting relaxation. A counter-example might be a phenomenon known as "relaxation-induced anxiety," where the biofeedback displays initiate or exacerbate anxiety in certain users during the relaxation training (Heide and Borkovec, 1983). Fortunately, our review shows that insights from HCI research have been used to address user experience and engagement by exploring new forms of biofeedback, such as musical displays (Yokoyama et al., 2002; Morarend et al., 2011; Vidyarthi and Riecke, 2013; Harris et al., 2014; Bhandari et al., 2015), ambient light displays (Snyder et al., 2015), VR displays (Gaggioli et al., 2014; van Rooij et al., 2016), and immersive physical environments (Adelbach et al., 2012). These new interfaces offer users a more comfortable and relaxing condition for biofeedback training. However, they may also require more effort for users to learn about and adapt to them.

\section{Discussion of the Use of Biofeedback}

As documented in Table 1, most current biofeedback applications still rely on a pre-scheduled training plan or program. Although a growing number of personal biofeedback devices can be used in daily life with no need for the assistance of a therapist, they were still used in the same way as in clinical training programs. For instance, college students completed an HRV biofeedback training program (20 min per day, 5 days per week) in a dedicated office space (Henriques et al., 2011). Manufacturing operators performed five biofeedback training sessions $(20 \mathrm{~min}$ ) once a week in a special training room at their factory (Sutarto et al., 2012). Some training programs involve a stress-induction session before the biofeedback session. Users first perform a mentally challenging task to induce a stress response and then learn to control and reduce that stress by means of self-regulation.

Biofeedback training may take longer (e.g., 3 months in McCraty et al., 2009) or even a very short time (e.g., 3 min in Yokoyama et al., 2002), depending on the training goal. On the one hand, biofeedback systems can help users acquire and enhance self-regulation skills. For this purpose, biofeedback techniques are often used in a multi-session training program lasting from 3 days (Bouchard et al., 2012) to 3 months (McCraty et al., 2009). On the other hand, biofeedback system can also facilitate relaxation, during which the biofeedback enables users to be aware of their bodily activities and regulate their physiology in a positive direction, e.g., by moderating arousal or enhancing HRV. In such cases, biofeedback can be used in a one-off relaxation session, which usually takes 5-30 min (Moraveji et al., 2012; Wells et al., 2012; Peira et al., 2014; Lee and Finkelstein, 2015).

The principles and practice of biofeedback training have been proposed for clinicians (Basmajian, 1979, 1981). However, most studies have not investigated the optimum duration of biofeedback and the efficiency of learning skills and practicing relaxation. We did not find any suggestions and guidelines for the duration of biofeedback training for everyday stress management. As this is beyond the scope of this article, we suggest that future research could investigate the relationship between the duration, frequency, and number of biofeedback training sessions and its efficiency, such as what would be the minimum length of a biofeedback session and what period would be most efficient/effective? From another perspective, beyond program-based regular biofeedback training, we believe the potential of biofeedback might be achieved through casual use. We think a stressful situation in real life presents the best opportunity to learn how to control stress. Biofeedback can be envisioned as a small "bio-mirror" that users can grab from their pocket at any time to "check" their stress level and manage the stress.

\section{Discussion of the Evaluation Approaches for Biofeedback in Everyday Stress Management}

As shown in Table 4, validation of biofeedback applications depends mainly on two types of metrics: physiological and psychological measures. The physiological data measured for biofeedback can also be stored and further analyzed to generate metrics for use in evaluation. In most evaluations, physiological measures are required to validate the biofeedback training comprehensively. For instance, to evaluate the effectiveness of an HRV biofeedback system for stress management, blood pressure, and salivary cortisol level (Lemaire et al., 2011) or respiration rate 
(Prinsloo et al., 2013) are measured. In these cases, users have to wear more biosensors, which limit evaluations to lab settings.

The psychological measures are often collected by applying a survey method involving standardized questionnaires. Users usually complete these surveys before and after a session of biofeedback training. Our review shows that most of these evaluations were conducted in laboratory settings and focused on the effectiveness of biofeedback systems or training programs. To gain more insights into everyday biofeedback design, we suggest a long-term field evaluation that combines quantitative with qualitative methods.

Other researchers have also suggested the need for a transition from laboratory study to field research (e.g., Lazarus, 2000) in order to better understand people's stress and coping paradigm. Field evaluations involve collecting users' data outside a laboratory or a training room, where the researchers may monitor, interview, and observe them in a familiar environment. Long-term physiological measurements may offer more robust and reliable metrics which reflect a users' real stress levels. In addition, interviews during field evaluations can provide highquality data about user experience because interviewers can adjust their questions based on specific situations to clarify issues or acquire further insights. Moreover, field evaluation makes it possible to observe users' behavior, e.g., when and how they use biofeedback devices.

\section{A New Biofeedback Paradigm Presents New Challenges}

Based on this review, it appears that a new biofeedback paradigm beyond traditional clinical applications is emerging. This is also creating new demands and challenges. Computer-mediated self-use is gradually replacing therapist-mediated biofeedback training. In such cases, users have to perceive and interpret feedback themselves. For long-term use, the systems also need to provide a good user experience and motivate users to engage further in biofeedback training. We believe that these challenges might be addressed by applying new interactive technologies and $\mathrm{HCI}$ principles in biofeedback systems.

New interactive technologies may help biofeedback better blend into an everyday context, improving its accessibility, usability, and comfort. For instance, HRV biofeedback using ambient light could improve a user's awareness of stress and trigger behavioral conditioning, such as deep breathing (Yu et al., 2017). In home environments, biofeedback can be displayed by a Chinese ink painting projected on the wall (Zhu et al., 2013) or with a shape-changing interface integrated into the furniture in the living room as part of the interior design (Feijs and Delbressine, 2017). An increasing number of everyday objects are being redesigned as handy biofeedback tools so that the users can use them more easily at any time. For instance, users can take a BioFidget out of their pocket and practice deep breathing when they feel stressed (Liang et al., 2018).

As Internet of Things (IoT) technology improves, we see that emerging IoT systems can serve as a new platform for biofeedback. For instance, we envision a "biofeedback mode" in future smart homes. Inhabitants' physiological data can be sensed unobtrusively. The collected multiple personal data are then analyzed using deep-learning techniques that generate more useful information, not only about the state of physiology and health but also about the behaviors and lifestyle that lead to that state. This information can be presented to users through IoT devices that are distributed in the environment, thus both improving their awareness of physiology and further assisting them to regulate it in a healthy direction. To our knowledge, there has not been any research looking at integrating IoT and biofeedback technologies. We suggest that this might be a promising direction for future research.

Moreover, we suggest using well-established HCI theories in the design of biofeedback which could enhance engagement as well as the user experience. For instance, when biofeedback is used to improve users' awareness of stress at work, we suggest an interesting direction of peripheral biofeedback, which has been explored preliminarily by Moraveji et al. (2011). As people's attention is almost fully occupied with work tasks, biofeedback displays should not become too "loud" and thus become the center of attention because this might cause a sense of burden or pressure. In line with the vision of Calm Technology (Weiser and Brown, 1997), we believe that leveraging human attention abilities in peripheral interactions (Bakker et al., 2015) will support biofeedback-enabled self-awareness and thus be more effective for stress management in the context of today's busy lifestyles. Acquiring self-regulation skills through biofeedback is not easy. It takes time and practice. So how to encourage users to engage in biofeedback training is an issue that deserves study. We suggest combining biofeedback with persuasive technology (Intille, 2004). And we believe that the ubiquitous biosensing and interactive technologies in everyday environments that are described in this article make it feasible to provide persuasive biofeedback at the right time and place so that users are more likely to change their behavior and practice self-regulation.

\section{CONCLUSION}

The aim of this review was to provide a reference for other researchers and designers so that they can better understand the current status of biofeedback technologies and their application for stress management. The results indicate that HRV and multimodal biofeedback systems are most commonly used and screen-based visual displays are the most common biofeedback interfaces. Biofeedback applications are mainly based on a pre-scheduled training routine and mostly assessed in laboratory settings, using both physiological and psychological measurements. This review also reveals several challenges arising in biofeedback for everyday stress management, including accessibility, usability, comfort, engagement, and user experience. We expect that new HCI technologies and theories will help meet these challenges. Moreover, we suggest a long-term field evaluation method that combines quantitative with qualitative methods in order to better understand the effects of biofeedback in everyday use, and how it influences users' behavior and lifestyle when they engage in stress management. 


\section{AUTHOR CONTRIBUTIONS}

$\mathrm{BY}, \mathrm{MF}, \mathrm{JH}$, and LF conceived and planned the literature review. BY and QW carried out the database search, abstract screening, and selection of the final articles for review. $\mathrm{JH}$,

\section{REFERENCES}

Abdelrahman, Y., Velloso, E., Dingler, T., Schmidt, A., and Vetere, F. (2017). "Cognitive heat: exploring the usage of thermal imaging to unobtrusively estimate cognitive load," in Proceedings of the ACM on Interactive, Mobile, Wearable and Ubiquitous Technologies, (New York, NY), 33.

Adelbach, H. S., Irune, A., Kirk, D. B., Glover, K., and Brundell, P. (2012). ExoBuilding: physiologically driven adaptive architecture. ACM Trans. Comput. Hum. Interact. 19, 1-22. doi: 10.1145/2395131.2395132

Adib, F., Mao, H., Kabelac, Z., Katabi, D., and Miller, R. C. (2015). "Smart homes that monitor breathing and heart rate," in Proceedings of the 33rd Annual ACM Conference on Human Factors in Computing Systems (CHI'15), (Seoul) 837-846.

Al Osman, H., Dong, H., and El Saddik, A. (2016). Ubiquitous biofeedback serious game for stress management. IEEE Access. 4, 1274-1286. doi: 10.1109/ACCESS.2016.2548980

Al Rihawi, R. G., Ahmed, B., and Gutierrez-Osuna, R. (2014). "Dodging stress with a personalized biofeedback game," in Proceedings of the First ACM SIGCHI Annual Symposium on Computer-Human Interaction in Play (CHI PLAY'14) (Toronto, ON), 399-400.

Alvarsson, J. J., Wiens, S., and Nilsson, M. E. (2010). Stress recovery during exposure to nature sound and environmental noise. Int. J. Environ. Res. Public Health 7, 1036-1046. doi: 10.3390/ijerph7031036

Arroyo-Palacios, J., and Slater, M. (2016). Dancing with physio: a mobile game with physiologically aware virtual humans. IEEE Trans. Affect. Comput. 7, 326-336. doi: 10.1109/TAFFC.2015.2472013

Bakker, S., van den Hoven, E., and Eggen, B. (2015). Peripheral interaction: characteristics and considerations. Pers. Ubiquit. Comput. 19, 239-254. doi: 10.1007/s00779-014-0775-2

Basmajian, J. V. (1979). Biofeedback: Principles and Practice for Clinicians. Oxford, UK: Williams \& Wilkins.

Basmajian, J. V. (1981). Biofeedback in rehabilitation: a review of principles and practices. Arch. Phys. Med. Rehabil. 62, 469-475.

Bhandari, R., Parnandi, A., Shipp, E., Ahmed, B., and Gutierrez-Osuna, R. (2015). "Music-based respiratory biofeedback in visually-demanding tasks," in Proceedings of the International Conference on New Interfaces for Musical Expression (Baton Rouge, LA), 78-82.

Blanchard, E. B., Young, L. D., Pines, M., Morris, S., Malloy, M. T., Andrews, L. M., et al. (1974). Clinical applications of biofeedback training. Arch. Gen. Psychiatry 30, 573-589. doi: 10.1001/archpsyc.1974.01760110003001

Bouchard, S., Bernier, F., Boivin, E., Morin, B., Robillard, G., Davydov, D. M., et al. (2012). Using biofeedback while immersed in a stressful videogame increases the effectiveness of stress management skills in soldiers. PLOS ONE 7:e36169. doi: 10.1371/journal.pone.0036169

Brown, B. B. (1977). Stress and the Art of Biofeedback. Oxford: Harper \& Row.

Burke, H. M., Davis, M. C., Otte, C., and Mohr, D. C. (2005). Depression and cortisol responses to psychological stress: a meta-analysis. Psychoneuroendocrinology 30, 846-856. doi: 10.1016/j.psyneuen.2005.02.010

Chittaro, L., and Sioni, R. (2014). Affective computing vs. affective placebo: study of a biofeedback-controlled game. Int. J. Hum. Comput. Stud. 72, 663-673. doi: 10.1016/j.ijhcs.2014.01.007

Cho, Y., Bianchi-Berthouze, N., and Julier, S. J. (2017), October. "DeepBreath: deep learning of breathing patterns for automatic stress recognition using lowcost thermal imaging in unconstrained settings," in 2017 Seventh International Conference on Affective Computing and Intelligent Interaction (ACII), (San Antonio, TX), 456-463.

Chrousos, G. P. (2009). Stress and disorders of the stress system. Nat. Rev. Endocrinol. 5, 374-381. doi: 10.1038/nrendo.2009.106

Chrousos, G. P., and Gold, P. W. (1992). The concepts of stress and stress system disorders. JAMA 267, 1244-1252. doi: 10.1001/jama.1992.03480090092034
MF, and LF verified the analytical methods. BY processed the literature data, performed the analysis, drafted the manuscript, and designed the figures. Both $\mathrm{MF}$ and $\mathrm{JH}$ contributed to the final version of the manuscript. LF supervised the study.

Constant, N., Douglas-Prawl, O., Johnson, S., and Mankodiya, K. (2015). "Pulse-glasses: an unobtrusive, wearable HR monitor with internet-of-things functionality," in IEEE 12th International Conference on Wearable and Implantable Body Sensor Networks (BSN), (Cambridge, MA), 1-5.

Cutshall, S. M., Wentworth, L. J., Wahner-Roedler, D. L., Vincent, A., Schmidt, J. E., et al. (2011). Evaluation of a biofeedback-assisted meditation program as a stress management tool for hospital nurses: a pilot study. J. Sci. Heal. 7, 110-112. doi: 10.1016/j.explore.2010.12.004

De Jonckheere, J., Ibarissene, I., Flocteil, M., and Logier, R. (2014). “A smartphone based cardiac coherence biofeedback system," in Proceedings of Annual International Conference of the IEEE Engineering in Medicine and Biology Society, (Chicago, IL), 4791-4794.

Dillon, A., Kelly, M., Robertson, I. H., and Robertson, D. A. (2016). Smartphone applications utilizing biofeedback can aid stress reduction. Front. Psychol. 7:832. doi: 10.3389/fpsyg.2016.00832

Droitcour, A., Lubecke, V., Lin, J., and Boric-Lubecke, O. (2001). “A microwave radio for doppler radar sensing of vital signs," in Proceedings of Microwave Symposium Digest, (Phoenix, AZ), 175-178.

Edvardsson, A., Ivarsson, A., and Johnson, U. (2012). Is a cognitive-behavioural biofeedback intervention useful to reduce injury risk in junior football players? J. Sports Sci. Med. 11, 331-338.

Esler, M., and Kaye, D. (2000). Sympathetic nervous system activation in essential hypertension, cardiac failure and psychosomatic heart disease. J. Cardiovasc. Pharmacol. 35, S1-S7. doi: 10.1097/00005344-20000000400001

Feijs, L., and Delbressine, F. (2017). "Calm technology for biofeedback: why and how?", in Proceedings of the Conference on Design and Semantics of Form and Movement - Sense and Sensitivity, (DeSForM 2017), (Eindhoven), 13-22.

Feijs, L., Kierkels, J., Van Schijndel, N. H., and Van Lieshout, M. (2013). "Design for relaxation during milk expression using biofeedback," in Proceedings of International Conference of Design, User Experience, and Usability (Las Vegas, NV), 494-503.

Frank, D. L., Khorshid, L., Kiffer, J. F., Moravec, C. S., and McKee, M. G. (2010) Biofeedback in medicine: who, when, why and how? Ment. Health Fam. Med. 7, 85-91.

Gaggioli, A., Pallavicini, F., Morganti, L., Serino, S., Scaratti, C., Briguglio, M., et al. (2014). Experiential virtual scenarios with real-time monitoring (interreality) for the management of psychological stress: a block randomized controlled trial. J. Med. Internet Res. 16:e167. doi: 10.2196/jmir.3235

Gaye, L., Mazé, R., and Holmquist, L. E. (2003). "Sonic City: the urban environment as a musical interface," in Proceedings of the 2003 Conference on New Interfaces for Musical Expression, (Montreal, QC), 109-115.

Glanz, M., Klawansky, S., and Chalmers, T. (1997). Biofeedback therapy in stroke rehabilitation: a review. J. R. Soc. Med. 90, 33-39. doi: 10.1177/014107689709000110

Goodie, J. L., and Larkin, K. T. (2006). Transfer of heart rate feedback training to reduce heart rate response to laboratory tasks. Appl. Psychophysiol. Biofeedback 31, 227-242. doi: 10.1007/s10484-006-9011-9

Greenhalgh, J., Dickson, R., and Dundar, Y. (2010). Biofeedback for hypertension: a systematic review. J. Hypertens. 28, 644-652. doi: 10.1097/HJH.0b013e3283370e20

Harris, J., Vance, S., Fernandes, O., Parnandi, A., and Gutierrez-Osuna, R. (2014). "Sonic respiration: controlling respiration rate through auditory biofeedback," in Proceedings of the Extended Abstracts of the 32nd Annual ACM Conference on Human Factors in Computing Systems (CHI EA'14) (New York, NY), 2383-2388.

Heide, F. J., and Borkovec, T. D. (1983). Relaxation-induced anxiety: Paradoxical anxiety enhancement due to relaxation training. J. Consult. Clin. Psychol. $51: 171$. 
Henriques, G., Keffer, S., Abrahamson, C., and Horst, S. J. (2011). Exploring the effectiveness of a computer-based heart rate variability biofeedback program in reducing anxiety in college students. Appl. Psychophysiol. Biofeedback 36, 101-112. doi: 10.1007/s10484-011-9151-4

Intille, S. S. (2004). A new research challenge: persuasive technology to motivate healthy aging. IEEE Trans. Inform. Technol. Biomed. 8, 235-237. doi: 10.1109/TITB.2004.835531

Ishii, H. (2008). "Tangible bits: beyond pixels," in Proceedings of the 2nd International Conference on Tangible and Embedded Interaction (TEI'08) (Bonn).

Ishii, H., Wisneski, C., Brave, S., Dahley, A., Gorbet, M., Ullmer, B., et al. (1998). "ambientROOM: Integrating ambient media with architectural space," in Proceedings of the Conference Summary on Human Factors in Computing Systems (CHI'98) (Los Angeles, CA), 173-174.

Johnston, W., and Mendelson, Y. (2005). "Extracting heart rate variability from a wearable reflectance pulse oximeter," in Proceedings of the IEEE 31st Annual Northeast Bioengineering Conference (Hoboken, NJ), 1-2.

Khalfa, S., Bella, S. D., Roy, M., Peretz, I., and Lupien, S. J. (2003). Effects of relaxing music on salivary cortisol level after psychological stress. Ann. N.Y. Acad. Sci. 999, 374-376. doi: 10.1196/annals.1284.045

Kotozaki, Y., Takeuchi, H., Sekiguchi, A., Yamamoto, Y., Shinada, T., Araki, T., et al. (2014). Biofeedback-based training for stress management in daily hassles: an intervention study. Brain Behav. 4, 566-579. doi: 10.1002/brb3.241

Kudo, N., Shinohara, H., and Kodama, H. (2014). Heart rate variability biofeedback intervention for reduction of psychological stress during the early postpartum period. Appl. Psychophysiol. Biofeedback. 39, 203-211. doi: 10.1007/s10484-014-9259-4

Lande, R. G., Williams, L. B., Francis, J. L., Gragnani, C., and Morin, M. L. (2010). Efficacy of biofeedback for post-traumatic stress disorder. Complement. Ther. Med. 18, 256-259. doi: 10.1016/j.ctim.2010.08.004

Larkin, K. T., Zayfert, C., Abel, J. L., and Veltum, L. G. (1992). Reducing heart rate reactivity to stress with feedback generalization across task and time. Behav. Modificat. 16, 118-131. doi: 10.1177/01454455920161006

Lazarus, R. S. (2000). Toward better research on stress and coping. Am. Psychol. 55, 665-673. doi: 10.1037/0003-066X.55.6.665

Lee, J., and Finkelstein, J. (2015). Evaluation of a portable stress management device. Stud. Health Technol. Inform. 208, 248-252. doi: 10.3233/978-1-61499-488-6-248

Lee, J., Kim, J. K., and Wachholtz, A. (2015). The benefit of heart rate variability biofeedback and relaxation training in reducing trait anxiety. Korean J. Health Psychol. 20, 391-408.

Lehrer, P. M., Vaschillo, E., and Vaschillo, B. (2000). Resonant frequency biofeedback training to increase cardiac variability: rationale and manual for training. Appl. Psychophysiol. Biofeedback. 25, 177-191. doi: 10.1023/A:1009554825745

Lemaire, J. B, Wallace, J. E., Lewin, A. M., De Grood, J., and Schaefer, J. P. (2011). The effect of a biofeedback-based stress management tool on physician stress: a randomized controlled clinical trial. Open Med. 5:e154.

Lewis, G. F., Hourani, L., Tueller, S., Kizakevich, P., Bryant, S., Weimer, B., et al. (2015). Relaxation training assisted by heart rate variability biofeedback: implication for a military predeployment stress inoculation protocol. Psychophysiology 52, 1167-1174. doi: 10.1111/psyp. 12455

Liang, R. H., Yu, B., Xue, M., Hu, J., and Feijs, L. M. (2018). "BioFidget: biofeedback for respiration training using an augmented fidget spinner," in Proceedings of the 2018 CHI Conference on Human Factors in Computing Systems, (Montreal, QC), 613. doi: 10.1145/3173574.3174187

Lloyd, C., Smith, J., and Weinger, K. (2005). Stress and diabetes: a review of the links. Diab. Spectr. 18, 121-127. doi: 10.2337/diaspect.18.2.121

Lundqvist, L.-O., Carlsson, F., Hilmersson, P., and Juslin, P. N. (2009). Emotional responses to music: experience, expression, and physiology. Psychol. Music 37, 61-90. doi: 10.1177/0305735607086048

Matuszek, T., and Rycraft, J. R. (2003). Using biofeedback to enhance interventions in schools. J. Technol. Hum. Services 21, 31-56. doi: 10.1300/J017v21n01_03

McCraty, R., Atkinson, M., Lipsenthal, L., and Arguelles, L. (2009). New hope for correctional officers: an innovative program for reducing stress and health risks. Appl. Psychophysiol. Biofeedback 34, 251-272. doi: 10.1007/s10484-0099087-0
Meier, N. F., and Welch, A. S. (2016). Walking versus biofeedback: a comparison of acute interventions for stressed students. Anxiety Stress Coping 29, 463-478. doi: 10.1080/10615806.2015.1085514

Moher, D., Liberati, A., Tetzlaff, J., Altman, D. G., and The PRISMA Group. (2009). Preferred reporting items for systematic reviews and meta-analyses: the PRISMA statement. PLoS Med. 6:e1000097. doi: 10.1371/journal.pmed.1000097

Moore, N. C. (2000). A review of EEG biofeedback treatment of anxiety disorders. Clin. EEG Neurosci. 31, 1-6. doi: 10.1177/155005940003100105

Morarend, Q. A., Spector, M. L., Dawson, D. V., Clark, S. H., and Holmes, D. C. (2011). The use of a respiratory rate biofeedback device to reduce dental anxiety: an exploratory investigation. Appl. Psychophysiol. Biofeedback 36, 63-70. doi: 10.1007/s10484-011-9148-z

Moraveji, N., Adiseshan, A., and Hagiwara, T. (2012). "BreathTray: augmenting respiration self-regulation without cognitive deficit," in Proceedings of the 2012 ACM Annual Conference Extended Abstracts on Human Factors in Computing Systems Extended Abstracts - (CHI EA'12) (San Jose, CA), 2405-2410.

Moraveji, N., Olson, B., Nguyen, T., Saadat, M., Khalighi, Y., Pea, R., et al. (2011). "Peripheral paced respiration: influencing user physiology during information work," in The 24th Annual ACM Symposium on User Interface Software and Technology (Santa Barbara, CA), 423-428.

Munafò, M., Patron, E., and Palomba, D. (2016). Improving managers psychophysical well-being: effectiveness of respiratory sinus arrhythmia biofeedback. Appl. Psychophysiol. Biofeedback 41, 129-139. doi: 10.1007/s10484-015-9320-y

Nestoriuc, Y., Martin, A., Rief, W., and Andrasik, F. (2008). Biofeedback treatment for headache disorders: a comprehensive efficacy review. Appl. Psychophysiol. Biofeedback 33, 125-140. doi: 10.1007/s10484-008-9060-3

Padgett, D. A., and Glaser, R. (2003). How stress influences the immune response. Trends Immunol. 24, 444-448. doi: 10.1016/S1471-4906(03) 00173-X

Parnandi, A., Ahmed, B., Shipp, E., and Gutierrez-Osuna, R. (2013). "Chill-out: relaxation training through respiratory biofeedback in a mobile casual game," in Proceedings of International Conference on Mobile Computing, Applications, and Services, (Paris), 252-260.

Paul, M., and Garg, K. (2012). The effect of heart rate variability biofeedback on performance psychology of basketball players. Appl. Psychophysiol. Biofeedback 37, 131-144. doi: 10.1007/s10484-012-9185-2

Peira, N., Fredrikson, M., and Pourtois, G. (2014). Controlling the emotional heart: heart rate biofeedback improves cardiac control during emotional reactions. Int. J. Psychophysiol. 91, 225-231. doi: 10.1016/j.ijpsycho.2013. 12.008

Poh, M.-Z., Kim, K., Goessling, A. D., Swenson, N. C., and Picard, R. W. (2009). "Heartphones: sensor earphones and mobile application for non-obtrusive health monitoring," in Proceedings of International Symposium on Wearable Computers (ISWC'09), (Linz), 153-154.

Porges, S. W. (1995). Cardiac vagal tone: a physiological index of stress. Neurosci. Biobehav. Rev. 19, 225-233. doi: 10.1016/0149-7634(94)00066-A

Prinsloo, G. E., Derman, W. E., Lambert, M. I., and Laurie Rauch, H. G. (2013). The effect of a single session of short duration biofeedback-induced deep breathing on measures of heart rate variability during laboratory-induced cognitive stress: a pilot study. Appl. Psychophysiol. Biofeedback 38, 81-90. doi: 10.1007/s10484-013-9210-0

Pusenjak, N., Grad, A., Tusak, M., Leskovsek, M., and Schwarzlin, R. (2015). Can biofeedback training of psychophysiological responses enhance athletes sport performance? A practitioner's perspective. Phys. Sportsmed. 43, 287-299. doi: 10.1080/00913847.2015.1069169

Rasmussen, M. K., Pedersen, E. W., Petersen, M. G., and Hornbæk, K. (2012). "Shape-changing interfaces: a review of the design space and open research questions," in Proceedings of the 2012 ACM Annual Conference on Human Factors in Computing Systems - (CHI'12) (Austin, TX), 735-744.

Ratanasiripong, P., Kaewboonchoo, O., Ratanasiripong, N., Hanklang, S., and Chumchai, P. (2015). Biofeedback intervention for stress, anxiety, and depression among graduate students in public health nursing. Nurs. Res. Pract. 2015:160746. doi: 10.1155/2015/160746

Reiner, R. (2008). Integrating a portable biofeedback device into clinical practice for patients with anxiety disorders: results of a pilot study. Appl. Psychophysiol. Biofeedback 33, 55-61. doi: 10.1007/s10484-007-9046-6 
Saha, S., Dey, D., Bhattacharyya, I. M., and Das, A. (2015). "An investigation on biofeedback analysis and psychosomatic applications," in Proceedings of International Conference on Recent Developments in Control, Automation and Power Engineering (RDCAPE), (Noida), 38-43.

Sanchez, D. I., Collins, T., Stone, R., and Woolley, S. I. (2012). "Cellular phonebased biofeedback to treat physical and mental disorders," in Proceedings of IEEE 14th International Conference on E-Health Networking, Applications and Services (Healthcom), (Beijing), 411-414.

Sarabia-Cobo, C. M. (2015). Heart coherence: a new tool in the management of stress on professionals and family caregivers of patients with dementia. Appl. Psychophysiol. Biofeedback 40, 75-83. doi: 10.1007/s10484-015-9276-y

Scott, L. D., Hwang, W. T., and Rogers, A. E. (2006). The impact of multiple care giving roles on fatigue, stress, and work performance among hospital staff nurses. J. Nurs. Administr. 36, 86-95. doi: 10.1097/00005110-200602000-00007

Smith, S. A. (2014). Mindfulness-based stress reduction: an intervention to enhance the effectiveness of nurses' coping with work-related stress. Int. J. Nurs. Knowl. 25, 119-130. doi: 10.1111/2047-3095.12025

Snyder, J., Matthews, M., Chien, J., Chang, P. F., Sun, E., and Abdullah, S. (2015). "MoodLight : exploring personal and social implications of ambient display of biosensor data," in Proceedings of the 18th ACM Conference on Computer Supported Cooperative Work \& Social Computing, 143-153.

Streeter, C. C., Gerbarg, P. L., Saper, R. B., Ciraulo, D. A., and Brown, R. P. (2012). Effects of yoga on the autonomic nervous system, gamma-aminobutyric-acid, and allostasis in epilepsy, depression, and post-traumatic stress disorder. Med. Hypotheses 78, 571-579. doi: 10.1016/j.mehy.2012.01.021

Strunk, K. K., Sutton, G. W., and Burns, N. S. (2009). Beneficial effects of accurate and false brief biofeedback on relaxation. Percept. Motor Skills 109, 881-886. doi: 10.2466/pms.109.3.881-886

Sutarto, A. P., Wahab, M. N., and Zin, N. M. (2012). Resonant breathing biofeedback training for stress reduction among manufacturing operators. Int. J. Occup. Saf. Ergon. 18, 549-561. doi: 10.1080/10803548.2012.11076959

Sztajzel, J. (2004). Heart rate variability: a noninvasive electrocardiographic method to measure the autonomic nervous system. Swiss Med. Weekly 134, $35-36$.

Tang, Y. Y., Ma, Y., Fan, Y., Feng, H., Wang, J., Feng, S., , et al. (2009). Central and autonomic nervous system interaction is altered by short-term meditation. Proc. Natl. Acad. Sci. U.S.A. 106 , 8865-8870. doi: 10.1073/pnas.0904031106

Turk, D. C., Meichenbaum, D. H., and Berman, W. H. (1979). Application of biofeedback for the regulation of pain: a critical review. Psychol. Bull. 86, 1322-1338. doi: 10.1037/0033-2909.86.6.1322

van der Zwan, J. E., de Vente, W., Huizink, A. C., Bögels, S. M., and de Bruin, E. I. (2015). Physical activity, mindfulness meditation, or heart rate variability biofeedback for stress reduction: a randomized controlled trial. Appl. Psychophysiol. Biofeedback. 40, 257-268. doi: 10.1007/s10484-015-9293-x

van Rooij, M., Lobel, A., Harris, O., Smit, N., and Granic, I. (2016). "DEEP:a biofeedback virtual reality game for children at-risk for anxiety," in Proceedings of the 2016 CHI Conference Extended Abstracts on Human Factors in Computing Systems (CHI EA'16) (San Jose, CA), 1989-1997.
Vidyarthi, J., and Riecke, B. E. (2013). "Mediated meditation: cultivating mindfulness with sonic cradle," in Proceedings of CHI'13 Extended Abstracts on Human Factors in Computing Systems, 2305-2314.

Weiser, M., and Brown, J. S. (1997). “The coming age of calm technology," in Beyond Calculation, (New York, NY: Springer) 75-85.

Wells, R., Outhred, T., Heathers, J. A., Quintana, D. S., Kemp, A. H., Ostwald, P. F., et al. (2012). Matter over mind: a randomised-controlled trial of single-session biofeedback training on performance anxiety and heart rate variability in musicians. PLOS ONE 7:e46597. doi: 10.1371/journal.pone.0 046597

Whited, A., Larkin, K. T., and Whited, M. (2014). Effectiveness of emWave biofeedback in improving heart rate variability reactivity to and recovery from stress. Appl. Psychophysiol. Biofeedback 39, 75-88. doi: $10.1007 / \mathrm{s} 10484-014-9243-\mathrm{z}$

Wu, W., Gil, Y., and Lee, J. (2012). Combination of wearable multi-biosensor platform and resonance frequency training for stress management of the unemployed population. Sensors 12, 13225-13248. doi: 10.3390/s121 013225

Wu, W., Zhang, H., Pirbhulal, S., Mukhopadhyay, S. C., and Zhang, Y. T. (2015). Assessment of biofeedback training for emotion management through wearable textile physiological monitoring system. IEEE Sens. J. 15, 7087-7095. doi: 10.1109/JSEN.2015.2470638

Yokoyama, K., Ushida, J., Sugiura, Y., Mizuno, M., Mizuno, Y., and Takata, K. (2002). Heart rate indication using musical data. IEEE Trans. Biomed. Eng. 49, 729-733. doi: 10.1109/TBME.2002.1010857

Yu, B., Funk, M., Hu, J., and Feijs, L. (2017). "StressTree: a metaphorical visualization for biofeedback-assisted stress management," in Proceedings of the 2017 Conference on Designing Interactive Systems (DIS'17), (Edinburgh), 333-337.

Zhu, B., Kürth-Landwehr, S., and Corbi, V. G. (2013). "YU: an artistic exploration of interface design for home healthcare," in Proceedings of the 8th International Conference on Tangible, Embedded and Embodied Interaction - TEI'14 (ACM Press), 332-334.

Zhu, Q., Kong, X.-L., and Xie, Y. (2012). "The influence of biofeedback on respiratory training effect," in Proceedings of 2012 International Conference on Systems and Informatics (ICSAI2012), (Yantai), 1067-1071.

Conflict of Interest Statement: The authors declare that the research was conducted in the absence of any commercial or financial relationships that could be construed as a potential conflict of interest.

Copyright (c) 2018 Yu, Funk, Hu, Wang and Feijs. This is an open-access article distributed under the terms of the Creative Commons Attribution License (CC BY). The use, distribution or reproduction in other forums is permitted, provided the original author(s) and the copyright owner(s) are credited and that the original publication in this journal is cited, in accordance with accepted academic practice. No use, distribution or reproduction is permitted which does not comply with these terms. 